\title{
Bonneau Identities
}

\author{
Hermès Bélusca-Maïto, Amon llakovac*, Marija Mađor-Božinović \\ Department of Physics, Faculty of Sciences, University of Zagreb, \\ Bijenička cesta 32, HR-10000 Zagreb, Croatia \\ E-mail: hbelusca@phy.hr, ailakov@phy.hr, mmadjor@phy.hr
}

\section{Dominik Stöckinger}

Institut für Kern- und Teilchenphysik, TU Dresden,

DE-01069 Dresden, Germany

E-mail: Dominik. Stoeckingeretu-dresden.de

The Bonneau identities are a very convenient tool for e.g. restoring BRST symmetry and deriving renormalization group equations in content of chiral gauge theories. The background for the Bonneau identities is Breitenlohner-Maison-'t Hooft-Veltman dimensional regularization scheme which is reviewed here with special emphasis to bridge the notational differences between the Breitenlohner-Maison and the Bonneau papers and identifying the notions in these references. The Bonneau identities are rederived but for a general theory and reexpressed in terms of the effective action, establishing the bridge to the expressions in the Martin-Sanchez-Ruiz reference. Several new interpretations of lemmas, theorems and notions are given.

Preprint number: ZTF-EP-20-07

PACS : $11.10 . \mathrm{Gh}, 11.30 . \mathrm{Rd}, 11.30 .-\mathrm{j}$

Corfu Summer Institute 2019 "School and Workshops on Elementary Particle Physics and Gravity" (CORFU2019)

31 August - 25 September 2019

Corfu, Greece

${ }^{*}$ Speaker. 


\section{Introduction}

Although the Bonneau identities $[1,2]$ are not widely used in the physical community, they have several important applications. In combination with the action principles $[3,4,5,6]$ they lead to a very convenient method for finding the finite counterterms in the framework of the axiomatically consistent Breitenlohner-Maison-t'Hooft-Veltman (BMHV) scheme [5, 7], which may lead to significant shortcut of calculations, usually performed in a conventional way using the SlavnovTaylor identities $[8,9,10]$. Second, using the algebraic renormalization techniques they lead to the method for finding the renormalization group equations $[1,2,6]$ which do use the renormalized effective action only.

The intention of this proceedings is to give an introduction to the Bonneau identities and the background for it, namely the BMHV scheme approach to the dimensional renormalization. The idea is to present the material in a consecutive way, and with lot of comments when argumenting the steps, which are not always or not obviosly written in the original articles.

The structure of the article is as follows. In the second section we give the overview of terms and notions for the BMHV scheme and list all basic statements, lemmas and theorems from the Breitenlohner-Maison (BM) paper which are used in derivation of the Bonneau identities. A special emphasis is given to the BM Lemma 5 and Proposition 3 [5]. In the third section the basic notions needed for deriving the Bonneau identities are listed. The basic Bonneau identity and the Bonneau identity for the trace anomaly are rederived with special emphasis to the application of the BM Proposition 3 and Lemma 5, as well as the notions used in the steps used to derive these identities. In the third section we also shortly describe the application of the Bonneau identities for restoring the BRST symmetry of a non-Abelian chiral gauge theory up to the essential anomalies [6], and do express the final results for amplitudes in terms of the corresponding effective action. The fourth section is the conclusion in which we stress the points which we emphasized to make the notion and derivation of the Bonneau identities more tractable for phenomenologists.

\section{Terms and Notions on Graphs and Amplitudes and Renormalization}

The Bonneau identities are defined using the Breitenlohner-Maison-t'Hooft-Veltman (BMHV) dimensional remormalization scheme [5, 7]. The basic notions, theorems and lemmas for the BMHV are given in this section.

\subsection{The Breitenlohner-Maison (BM) definition of the amplitude which is defined in terms of a set of graph-theory terms.}

2.1.1. Graph notions: We list here the basic graph notions together with the references where they are defined :

Definition of a (Feynman) graph [11, 5]: its vertices $\mathscr{V}^{G}=\left\{V_{i}, i=1, \ldots, M\right\}$, lines $\mathscr{L}^{G}=\left\{\ell_{\ell}, \ell=\right.$ $1, \ldots, L\}$, number of loops $h_{G}$ and incidence matrix $e$ [11, 5]; Euler formula [12];

Types of graphs: empty graph [13], nonempty graph [5], trivial graph [13], nontrivial graph [5], connected graph [5], subgraph [14,13], proper subgraph [11, 5, 13], conectivity components (maximal connected components) of a graph, graph $G$ with set of lines $\mathscr{L}$ removed $G-\mathscr{L}[11,5]$, one-particle irreducible (1PI, proper) (sub)graph [15, 5], maximal 1PI subgraph (1PI connectivity 
components) of a graph [12, 5], non-overlapping (sub)graphs [14, 15, 5], overlapping (sub)graphs [14, 15, 5], reduced graph $G / H[11,5]$, tree [11, 12, 15, 5], chord [11], 2-tree [11, 12, 5], complement of 2-tree [11];

Forests of a graph $G$ : definition of forest $[14,16]$, normal forests, full forests [14, 15, 16], maximal forests [14, 5], restricted forests [14].

2.1.2. Amplitude: The BM amplitude [5] in the $D$-dimensional space is initially defined in the coordinate space, including the definitions of vertex operators $X_{i}\left(-i \partial / \partial x_{i}\right)$ and differential operators defining numerators for propagators $Z_{\ell}\left(-i \partial / \partial u_{\ell}\right)$ having masses $\left\{m_{\ell}\right\}=\underline{m}$, where $\underline{u}=\left\{u_{\ell}\right\}$ are auxiliary variables of mass dimension -1 , introduced to project out momenta of internal lines. Propagators are written in the exponential form using Schwinger parameters $\underline{\alpha}=\left\{\alpha_{\ell}\right\}$, of mass dimension -2 . The amplitude is then Fourier-transformed. This leads to several effects. Namely, the coordinate dependence of the exponent, its Gaussian structure and the structure of the incidence matrix lead to:

i. the conservation of the total external momentum;

ii. the dependence of the amplitude: on the differences of external momenta (except in the vertex factors), on the diagonal matrix of the Schwinger parameters $\alpha$, on the reduced incidence matrix $e_{E}^{k}=\left(\{e\}_{\ell i}\right)^{k}$ (of the mass dimension 0) with a $k-t h$ column deleted in $e$, and the reduced set of external momenta $p_{E}^{k}=\left\{p_{E}\right\}^{k}$ with the $k$-th momentum deleted, on the matrix $\left(A_{E}^{k}\right)^{-1}=$ $\left(\left(e_{E}^{k}\right)^{T} \alpha^{-1} e_{E}^{k}\right)^{-1}$ and on the determinant of a matrix $M$ defined below $d(\alpha)$.

All quantities in the amplitude are independent on a choice of $k$, specifically $d(\alpha)$ and $\left(p_{E}^{k}\right)^{T}\left(A_{E}^{k}\right)^{-1} p_{E}^{k}$ do not depend on $k$. The quantities $d(\alpha)$ and $\left(A_{E}^{k}\right)^{-1}$ can be expressed in terms of minors $A(k ; k)$ and $A(k i ; k j)$ of the matrix $A=e^{T} \alpha^{-1} e$, obtained by deleting a $k$-th row and column, and by deleting a $k$-th and a $i$-th rows and the $k$-th and a $j$-th columns in $A$, respectively, and are related to the Symanzik polynomials $[17,5]$ which characterize an amplitude of any loop diagram.

The final expression for the amplitude for a graph $G$ is

$$
\mathscr{T}_{G}(\underline{p})=\left.\lim _{\varepsilon \rightarrow 0} \hbar^{h_{G}-1}(2 \pi)^{D / 2} \delta^{D}\left(\sum p_{i}\right)\left(\frac{i}{2}\right)^{-D h_{G} / 2} \int \prod_{\ell \in \mathscr{L}_{G}} d \alpha_{\ell} I_{\varepsilon}(\underline{p}, \underline{u}, \underline{\beta})\right|_{\underline{u}=0}
$$

where:

$$
\begin{aligned}
I_{\varepsilon}(\underline{p}, \underline{u}, \underline{\alpha}) & =d(\underline{\alpha})^{-D / 2} \prod_{i \in \mathscr{L}_{G}} X_{i}\left(p_{i},-i \frac{\partial}{\partial u_{\ell}}\right) \prod_{\ell \in \mathscr{L}_{G}} Z_{\ell}\left(-i \frac{\partial}{\partial u_{\ell}}\right) \exp i W\left(\underline{p}_{E}, \underline{u}, \underline{\beta}\right), \\
W\left(\underline{p}_{E}, \underline{u}, \underline{\alpha}\right) & =V\left(\underline{p}_{E}, \underline{u}, \underline{\alpha}\right)-\underline{\alpha}\left(\underline{m}^{2}-i \varepsilon\right), \\
V\left(\underline{p}_{E}, \underline{u}, \underline{\alpha}\right) & =\left(\underline{p}_{E}^{T}, \underline{u}^{T}\right)\left(\begin{array}{rr}
0 & -2 e_{E}^{T} \\
-2 e_{E} & -4 \alpha
\end{array}\right)^{-1}\left(\begin{array}{c}
\underline{p}_{E} \\
\underline{u}
\end{array}\right) \equiv\left(\underline{p}_{E}^{T}, \underline{u}^{T}\right) M^{-1}\left(\begin{array}{c}
\underline{p}_{E} \\
\underline{u}
\end{array}\right), \\
d(\underline{\alpha}) & =\operatorname{det} M /(-4)^{L},
\end{aligned}
$$

with the infinitesimal $\varepsilon$ defining the Feynman structure of the propagator denominators in the momentum space. It should be noted that due to the momentum conservation in the vertex operator $X_{i}$ one can make a replacement $p_{i} \rightarrow e_{i \ell}^{T} \partial / \partial u_{\ell}$.

2.2. Tensor and Dirac algebra [5]: In the BMHV scheme the $D$-dimensional space is understood 
to possess its 4-dimensional and $D-4$ dimensional sub-spaces. To these three (sub)spaces correspond metric tensors $g^{\mu v}, \bar{g}^{\mu v}$ and $\widehat{g}^{\mu v}$, by the action of which on the $D$-dimensional objects one defines objects living in all three spaces respectively, for example $\gamma^{\mu}$ matrices, covariant derivatives, vector fields. The $\gamma^{\mu}$ matrices satisfy Grassmann algebra in all three spaces. Nevertheless, the Levi-Civita symbol $\varepsilon^{\mu v \rho \sigma}$ and the $\gamma^{5}$ matrix are defined only in 4 dimensions. In the BMHV scheme $\gamma^{\mu}$ matrices do not anticommute with the $\gamma_{5}$ matrix. The 4-dimensional part of $\gamma_{\mu}$ matrices anticommute with $\gamma_{5}$, but their $D-4$ dimensional part commute with $\gamma_{5}$. This approach enables one to define a theory which is axiomatically correct in the sense of Hepp [18], and to reproduce the Adler-Bell-Jackiw anomaly $[19,20]$. The metric tensor $\widehat{g}^{\mu v}$, and all vector objects which might be written as a contraction corresponding to $D$ dimensional vector object with $\widehat{g}^{\mu v}$, are called evanescent.

Using the Dirac algebra rules of the BMHV scheme, every amplitude has to be reduced to its simplest form, the so called "normal form" [5]. The $D$-dimensional amplitude thus obtained is a meromorphic function of the complex variable $D$, which, between all the poles the amplitude has, has the poles at $D=4$, used to define counterterms for the amplitude.

\subsection{Labelled forests and their role in the renormalization procedure [5]:}

For a connected graph $G$ with 1PI components $G_{i}$, a maximal forest for $G, \mathscr{C}$ (or $\mathscr{C}_{G}$ ), is a maximal set of non-trivial non-overlapping 1PI subgraphs of $G$ (for 1PI diagram $G$ the number of maximal forests is equal to $h_{G}$, the number of loops of $G$ ). For any maximal forest $\mathscr{C}$ and any $H \in \mathscr{C}$ (therefore $H \subseteq G$ ) a set $\mathscr{M}(H)$ of maximal elements of $\mathscr{C}$ properly contained in $H$ (for $X \in \mathscr{M}(H)$ where $X \subset H)$ is defined. It is further used to define a set of complements of elements of $\mathscr{M}(H)$ with respect to $H, H / \mathscr{M}(H)=\{\bar{H} \in H / \mathscr{M}(H)\}$. For each $H \in \mathscr{C}, \bar{H}$ is chosen (there may be more choices of $\bar{H}$ for each maximal forest $\mathscr{C}_{G}$ of $G$ ). For each specific choice of $\bar{H}$ 's one defines a mapping $\sigma: \mathscr{C} \rightarrow \mathscr{L}_{G}$ such that $\sigma(H)=\mathscr{L}_{\bar{H}}=\{$ lines of $\bar{H}\}$. A pair $(\mathscr{C}, \sigma)_{G}$ is a labelled forest for $G$. To it is adjoined a subset of $\underline{\alpha}$-space of Schwinger parameters defined by

$$
\mathscr{D}(\mathscr{C}, \sigma)=\left\{\left(\alpha_{1}, \ldots, \alpha_{L}\right): \alpha_{\ell} \geq 0 \forall \ell \in G ; \alpha_{\ell} \leq \alpha_{\sigma(H)} .\right.
$$

In addition, maximal forests and labelled forests have the following properties [5]:

a. Any maximal forest $\mathscr{C}_{G}$ for $G$ is a disjoint union of maximal forests $\mathscr{C}_{G_{i}}$ for its 1PI components $G_{i}$.

b. Any maximal forest $\mathscr{C}_{G}$ for $G$ may be labelled.

c. For any 1PI subgraph $H$ there is a one-to-one correspondence between the labelled forest $(\mathscr{C}, \sigma)$ and pairs of $\left(\left(\mathscr{C}_{1}, \sigma_{1}\right),\left(\mathscr{C}_{1}, \sigma_{1}\right)\right)$ of labelled forests for $G / H$ and $H$.

d. Any maximal forest for $G$ has $h_{G}$ elements.

e. For any $\sigma\left(\mathscr{C}_{G}\right)$ of $G, G-\sigma\left(\mathscr{C}_{G}\right)$ is a tree in $G$. Thit means that for any choice of $\{\bar{H}\}$ 's for a given $\mathscr{C}$ of $G, \sigma(\mathscr{C})=\bigcup_{H} \mathscr{L}_{\bar{H}}$ forms a chord of $G$.

f. The union of all subsets of Schwinger parameters $\mathscr{D}(\mathscr{C}, \sigma)$ covers the whole space of Schwinger parameters of the amplitude for $G,\left\{\alpha_{\ell}: \alpha_{\ell} \geq 0 \forall \ell\right\}: \bigcup_{(C, \sigma)}=\left\{\alpha_{\ell}: \alpha_{\ell} \geq 0 \forall \ell\right\}$.

g. For $(\mathscr{C}, \sigma) \neq\left(\mathscr{C}^{\prime}, \sigma^{\prime}\right), \mathscr{D}(\mathscr{C}, \sigma) \cap \mathscr{D}\left(\mathscr{C}^{\prime}, \sigma^{\prime}\right)$ is a set of Lebesgue measure zero.

In conclusion, for a graph $G$, one finds all maximal forests $\mathscr{C}$. For each maximal forest one finds all sets of $\bar{H}$ lines which form chords of $G$ for a given maximal forest. To every such set is adjoined a mapping $\sigma$ and a subset of Schwinger parameters $\mathscr{D}(\mathscr{C}, \sigma)$. The introduced quantities satisfy the 
properties a.-g.

Using the property f. of labelled forests the amplitude becomes [5]

$$
\mathscr{T}_{G}=\lim _{\varepsilon \rightarrow 0} \mathscr{T}_{G, \varepsilon}=\lim _{\varepsilon \rightarrow 0} \int d \underline{\alpha} I_{G, \varepsilon}(\underline{p}, \underline{\alpha})=\lim _{\varepsilon \rightarrow 0} \sum_{(\mathscr{C}, \sigma)} \int_{\mathscr{D}(\mathscr{C}, \sigma)} d \underline{\alpha} I_{G, \varepsilon}(\underline{p}, \underline{\alpha}) .
$$

Using the Zimmermann forest formula $[14,15]$, assuming the action of the counterterm $C_{H}$ of each subgraph $H \subset G$ of $I_{G}$ is known (see subsection 2.6), and that $C_{H}$ is defined so that $[14,15]$ for any forest $F \in \mathscr{F}(\mathscr{F}=\{F\}$ is the set of all forests $F$ of $G)$ the relation $\int_{\mathscr{D}(\mathscr{C}, \sigma)} d \underline{\alpha} \prod_{H \in F}\left(-C_{H}\right) I_{G, \varepsilon}(\underline{p}, \underline{\alpha})=$ 0 unless $F \subseteq \mathscr{C}$ is fulfilled (if $F \nsubseteq \mathscr{C}$ then $C_{H} I_{G, \varepsilon}(\underline{p}, \underline{\alpha}) \in \mathscr{D}\left(\mathscr{C}^{\prime}, \sigma^{\prime}\right),\left(\mathscr{C}^{\prime}, \sigma^{\prime}\right) \neq(\mathscr{C}, \sigma)$, so the zero result is a consequence of property $\mathbf{g}$. of labelled forests), the renormalized amplitude reads

$$
\begin{aligned}
\mathscr{R}^{G} & =\lim _{\varepsilon \rightarrow 0} \mathscr{R}_{v, \varepsilon}^{G}=\lim _{\varepsilon \rightarrow 0} \sum_{(\mathscr{C}, \sigma)} \int_{\mathscr{D}(\mathscr{C}, \sigma)} d \underline{\alpha} \sum_{F \in \mathscr{F}} \prod_{H \in F}\left(-C_{H}\right) I_{G, \varepsilon}(\underline{p}, \underline{\alpha}) \\
& =\lim _{\varepsilon \rightarrow 0} \sum_{(\mathscr{C}, \sigma)} \int_{\mathscr{D}(\mathscr{C}, \sigma)} d \underline{\alpha} \prod_{H \in \mathscr{C}}\left(1-C_{H}\right) I_{G, \varepsilon}(\underline{p}, \underline{\alpha}) .
\end{aligned}
$$

The first line corresponds to the Zimmermann forest formula, while the second is its re-expression in terms of labelled forests [5]. Note that for each labelled forest the Dyson renormalization formula $[21,22]$ can be used.

2.4. Changes of variables: The variables $\alpha_{\ell}$ and the choice of the momenta $\underline{p}$ are not appropriate for extracting singularities.

2.4.1. Variables $(\mathbf{t}, \beta)$ : For this reason more appropriate variables $\left\{(\underline{t}, \underline{\beta})=\left(t_{H}, H \in \mathscr{C}\right), \ell \in \mathscr{L}_{G}^{\prime}=\right.$ $\left.\mathscr{L}_{G}-\sigma(\mathscr{C})\right\}$ and corresponding auxiliary variables $\zeta_{H}$ and $\xi_{H}$ are introduced for a labelled forest $\mathscr{D}(\mathscr{C}, \sigma)[5]$

$$
\alpha_{\ell}=\left\{\begin{array}{l}
\prod_{H \subseteq H^{\prime} \in \mathscr{C}} t_{H^{\prime}}^{2}=t_{H}^{2} \xi_{H}^{2}=\zeta_{H}^{2} \text { if } \ell=\sigma(H), H \in \mathscr{C} \\
\beta_{\ell} \zeta_{H}^{2} \text { if } \ell \in \mathscr{L}_{\bar{H}}^{\prime}, H \in \mathscr{C}
\end{array}\right.
$$

and taking $\beta_{\ell}=1$ for $\ell \in \sigma(H)$. All the new variables are dimensionless, except for $t_{G}$ which has mass dimension -1 . The variables assume the following values $0 \leq t_{G}<\infty, 0 \leq t_{H} \leq 1$ for $H \neq G$ and $0 \leq \beta_{\ell} \leq 1$ for $\ell \in \mathscr{L}^{\prime}$.

The transformation between $\underline{\alpha}$ and $\left(\underline{t}_{H}, \underline{\beta}_{H}\right)$ and the parameter region $\mathscr{D}(\mathscr{C}, \sigma)$ in terms of new variables are

$$
\begin{gathered}
d \underline{\alpha}=\prod_{\ell=1}^{L} d \alpha_{\ell}=\left(\prod_{H \in C} 2 t_{H} t_{H}^{2 L_{H}-1}\right)\left(\prod_{\ell \in \mathscr{L}_{G}^{\prime}} d \beta_{\ell}\right), \\
\mathscr{D}(\mathscr{C}, \sigma)=\left\{(\underline{t}, \underline{\beta}) \mid 0 \leq t_{G}<\infty ; 0 \leq t_{H} \leq 1 \text { for } H \neq G ; 0 \leq \beta_{\ell} \leq 1 \text { for } \ell \in \mathscr{L}_{G}^{\prime}\right\} .
\end{gathered}
$$

After changing the variables, the part of the amplitude (2.1) under the integral sign, and corresponding to the labelled forest $\mathscr{D}(\mathscr{C}, \sigma)$, reads

$\int_{\mathscr{D}(\mathscr{C}, \sigma)} d \underline{\alpha} I_{G, \varepsilon}(\underline{p}, \underline{\alpha})=\prod_{\ell \in \mathscr{L}_{G}^{\prime}} \int_{0}^{1} d \beta_{\ell}\left\{\prod_{H \in \mathscr{C}} \int_{0}^{\theta_{H}} \mu^{v} \frac{2 d t_{H}}{t_{H}} t_{H}^{2 L_{H}}\left\{d(\underline{\alpha})^{-D / 2} Z_{H}\left(-i \frac{\partial}{\partial u_{\ell}}\right) e^{i W(\underline{p}, \underline{u}, \underline{t}, \underline{\beta})}\right\}\right\}$, 
with $\theta_{G}=\infty, \theta_{H}=1$ for $H \neq G$, and $Z_{H}\left(-i \partial / \partial u_{\ell}\right)=\prod_{\ell \in \bar{H}} Z_{\ell}\left(-i \partial / \partial u_{\ell}\right) \prod_{i \in \bar{H}} X_{i}\left(i e_{i l}^{T} \partial / \partial u_{\ell}\right.$, $\left.-i \partial / \partial u_{\ell}\right)$. In content of the BMHV renormalization scheme, the regularization mass parameter/scale $\mu$ [23], was introduced in the integration measure by Bonneau [1]. Notice that $\underline{\alpha}$ and $\underline{u}$ are dimensionful parameters, and that $d(\alpha)=d(\alpha(\underline{t}, \beta))$ depends on all the $\underline{t}_{H}$ variables.

2.4.2. Dimensionless covariants $\tilde{\mathbf{q}}$, $\underline{\mathbf{u}}$ : The next step is to group external momenta of the graph $G$ into momenta of $\bar{H}$ of all its subsets $H$ which are defined by the mapping $H \rightarrow H / \mathscr{M}(H)=\bar{H}$ of $H$ 's in a maximal forest $\mathscr{C}$ of $G$ [5]. The mapping is linear and defines the momenta of each $\bar{H}$ in terms of linear independent momenta $\underline{p}=\left\{p_{i}, i=1, \ldots M-1\right\}$ of $G$, replacing the mometa of $H^{\prime} \in \mathscr{M}(H)$ by its sum and keeping other $H(\bar{H})$ momenta unchanged. The obtained momenta for $H$ are linearly dependent and one has to erase one of them to obtain the linearly independent set of momenta $q_{H}$. The set of all such momenta $q_{H}$ for all $H$ 's is $q=\left\{q_{\ell}\right\}=\left\{q_{H}, H \in \mathscr{C}\right\}=R p$. To obtain the correct expression for $V(\underline{p}, \underline{u}, \underline{\alpha})$ in Eq. (2.1) $e_{E}$ must transform accordingly, $e_{E} \rightarrow e_{E} R^{T}$. It is also convenient to decompose $\underline{u}=\left\{u_{\ell}, \ell \in \mathscr{L}_{\bar{H}}\right\}$ and $e_{E}$ to have the same $H$ substructure as $\underline{q}: \underline{u}=\left\{\underline{u}_{H}=\left\{u_{\ell}, \ell \in \mathscr{L}_{\bar{H}}\right\}, H \in \mathscr{C}\right\}, e_{E} R^{T}=\left\{e_{H H^{\prime}}, H, H^{\prime} \in \mathscr{C}\right\}$. In addition, in order to extract the $t_{H}$ variables from $d(\underline{\alpha})$, and to express $V(\underline{p}, \underline{u}, \underline{\alpha})$ from Eq. (2.1) in terms of dimensionless quantities, dimensionless momenta and variables are introduced [5]: $\underline{q}^{\tilde{q}}=\left\{\underline{\tilde{q}}_{H}=\underline{q}_{H} \zeta_{H}, H \in \mathscr{C}\right\}$, $\underline{\tilde{u}}=\left\{\underline{u}_{H}=\underline{u}_{H} / \zeta_{H}, H \in \mathscr{C}\right\}, \underline{\tilde{e}}=\left\{\tilde{e}_{H H^{\prime}}=\left(e_{E} R^{T}\right)_{H H^{\prime}} \zeta_{H} / \zeta_{H^{\prime}}\right\}$ in terms of which

$$
\begin{aligned}
& V(\underline{p}, \underline{u}, \underline{\alpha})=\left(\underline{\tilde{q}}^{T}, \underline{\tilde{u}}^{T}\right) \tilde{M}^{-1}\left(\begin{array}{l}
\underline{\tilde{q}} \\
\underline{\tilde{u}}
\end{array}\right), \quad \text { where } \quad \tilde{M}^{-1}=\left(\begin{array}{cc}
0 & -2 \tilde{e} \\
-2 \tilde{e}-4 \beta
\end{array}\right), \\
& d(\underline{\alpha})=\tilde{d}(\underline{\beta}, \underline{t}) \prod_{H \in \mathscr{C}} \zeta_{H}^{2 h_{\bar{H}}}=\tilde{d}(\underline{\beta}, \underline{t}) \prod_{H \in \mathscr{C}} t_{H}^{2 h_{H}}, \quad \text { where } \quad \tilde{d}_{H}=\operatorname{det} \tilde{M} /(-4)^{L} .
\end{aligned}
$$

Here $R$ is a square triangle matrix with unit values on its diagonal. Furthermore, $\tilde{e}=\left\{\tilde{e}_{H H^{\prime}}\right\}$ is equal to 0 if $H \nsupseteq H^{\prime}$, is equal to $e_{H H}$ if $H=H^{\prime}$, and gives information on how $H^{\prime}$ is contained in $H$ if $H^{\prime} \subset H$. Concerning $\tilde{M}$ and $\tilde{d}_{H}^{-1}$ they do not depend on $t_{G}$, and are analytic $C^{\infty}$ functions of the $\beta_{\ell}$ variables and the remaining $t_{H}$ variables.

2.5. Divergences: Now the divergences can be localized. The amplitude (2.7), obtained by inserting (2.8) into it, reads [5]

$$
\begin{aligned}
\int_{\mathscr{D}(\mathscr{C}, \sigma)} d \underline{\alpha} I_{G, \varepsilon}(\underline{p}, \underline{\alpha}) & \left.=\prod_{\ell \in \mathscr{L}_{G}^{\prime}} \int_{0}^{1} d \beta_{\ell}\left\{\prod_{H \in \mathscr{C}} \int_{0}^{\theta_{H}} \mu^{v} \frac{2 d t_{H}}{t_{H}} t_{H}^{v h_{H}-\omega_{H}} Z_{H}\left(-i \frac{\partial}{\partial \tilde{u}_{\ell}}\right) g_{\varepsilon}(\underline{q}, \underline{u}, \underline{t}, \underline{\beta}, v)\right\}\right\} \\
& =\prod_{H \in \mathscr{C}}\left\{\int d \mu_{H} \zeta^{v-\omega_{\bar{H}}} Z_{H}\left(-i \frac{\partial}{\partial \tilde{u}}\right)\right\} g(\underline{q}, \underline{u}, \underline{t}, \underline{\beta}, v)
\end{aligned}
$$

where $v=4-D ; \omega_{H}=4 h_{H}-2 L_{H}+r_{H}, r_{H}=\sum_{\mathscr{L}_{H}} r_{\ell}+\sum_{\mathscr{V}_{H}} v_{i}$ where $r_{\ell}$ and $v_{i}$ are numbers of momenta in the propagator numerators $Z_{\ell}$ and the vertex operators $X_{i}$ respectively; $\omega_{\bar{H}}=\omega_{H}-$ $\sum_{H^{\prime} \in \mathscr{M}(H)} \omega_{H^{\prime}} ; d \mu_{H}=2 \int_{0}^{\theta_{H}} \mu^{v} d t_{H} / t_{H} \int_{0}^{1} \prod_{\ell \in \mathscr{L}_{H}} ; g_{\varepsilon}(\underline{q}, \underline{u}, \underline{t}, \underline{\beta}, v)=\tilde{d}(\underline{\beta}, \underline{t})^{-D / 2} \exp i W(\underline{p}, \underline{u}, \underline{t}, \underline{\beta})$ is a function analytic in $v$ and exponentialy decreasing as $t_{G} \rightarrow \infty$.

The source of divergences are the functions $t_{H}^{v h_{H}-\omega_{H}-1}$. They have to be actually interpreted as distributions of the form $t_{+}^{\lambda}$, which correspond to meromorphic functions of $\lambda$ with simple poles at all negative integers $[24,5]$, specifically

$$
\left(t_{H}\right)_{+}^{v h_{H}-\omega_{H}-1}=(-)^{\omega_{H}} \delta^{\left(\omega_{H}\right)}\left(t_{H}\right) /\left(\omega_{H} ! v h_{H}\right)+\text { regular at } v=0
$$


where "regular at $v=0$ " represents a regular function at $v=0$, specified by its integral below, and the $\delta^{\left(\omega_{H}\right)}\left(t_{H}\right)$ is $\omega_{H}$-th derivative of the $\delta\left(t_{H}\right)$-distribution for which $\delta\left(t_{H}\right) / \omega_{H}$ ! $=0$ for $\omega_{H}<0$. Using (2.10) the $t_{H}$ integrals in (2.9) become equal to $[24,5]$

$$
\begin{gathered}
\int_{0}^{\theta_{H}} t_{H}^{v h_{H}-\omega_{H}-1} g_{\varepsilon}\left(., t_{H}, .\right)=\left.\frac{1}{\omega_{H} ! v h_{H}} \frac{d^{\omega_{H}} g_{\varepsilon}\left(., t_{H}, .\right)}{d t_{H}^{\omega_{H}}}\right|_{t_{H}=0}+\int_{0}^{\theta_{H}}(\ldots)_{r e g} d t_{H}, \\
\int_{0}^{\theta_{H}}(\ldots)_{r e g} d t_{H}=\int_{0}^{\theta_{H}} d t_{H} t_{H}^{\nu h_{H}-\omega_{H}-1}\left[g_{\varepsilon}\left(., t_{H}, .\right)-\sum_{k=0}^{\omega_{H}-1} \frac{t_{H}^{k}}{k !} g^{(k)}\left(., t_{H}=0, .\right)\right. \\
\left.-\theta\left(1-t_{H}\right)\left(\frac{t_{H}^{\omega_{H}}}{\omega_{H} !} g^{\left(\omega_{H}\right)}\left(., t_{H}=0, .\right)\right)\right] .
\end{gathered}
$$

The divergences of the amplitudes are used to define counterterms of the amplitude.

2.6. Counterterm: The definition of the counterterm [5] of a subgraph $H, C_{H}$, requires several notions: the algebra of the covariants on the graph $G: \mathscr{A}_{G}$, the operator that extracts the divergence (principal part) of an element of the algebra of covariants $C$, and the definition of the domain for $C_{H}: \mathscr{D}_{H}$. The algebra of covariants is generated by any linear combination of products of elements of $\left\{p_{i, H}\right\}_{i \in \mathscr{V}_{G}}$, elements of $\left\{u_{l, H}\right\}_{i \in \mathscr{L}_{G}}$ (see Eq (2.1)), and $\left\{\gamma^{\mu}\right\}$ matrices. The operator $C$ extracts from the normal form (NF) of each element $A \in \mathscr{A}_{G}$ its singular part according to the $M S$ scheme,

$$
C(A)=\text { singular part at } D=4 \text { of } \operatorname{NF}\left(\left.A\right|_{\underline{u}=0}\right) .
$$

The definition of the domain $\mathscr{D}_{H}$ of $C_{H}$ requires the introduction of two more notions. The first is the definition of a set $\mathscr{G}_{H}$ of triplets $\widehat{X}=\left(X, F_{X},(\mathscr{C}, \sigma)_{X}\right)$, where $X \subset G, F_{X}$ is a forest (any forest, in the sense of Zimmermann [14, 15]) of 1PI, pairwise disjoint proper subgraphs of $X, \mathscr{F}_{X}^{\prime}$ is set of all forests $F_{X}$, and $(\mathscr{C}, \sigma)_{X}$ is a labelled forest for $X / F_{X}$. The second is a set of functions $\mathscr{E}_{G}$ on $\mathscr{G}_{G}$ with values in $\mathscr{A}_{G}$ defined by $\mathscr{E}_{G}=\left\{f: \mathscr{G}_{G} \rightarrow \mathscr{A}_{G}\right\}$, where each $f(\widehat{X})$ depends only on variables $(\underline{p}, \underline{u})$ from $X / F_{X}$. The domain for $C_{H}$ is defined as a subset of functions of $\mathscr{E}_{G}$ defined on a specific set of triplets $\widehat{H}$ and polynomial form of $C(f(\widehat{X})), \mathscr{D}_{H}=\left\{f \in \mathscr{E}_{G} \mid\right.$ for all $\widehat{X}=\widehat{X}_{0} \equiv$ $\left(X, \emptyset,(\mathscr{C}, \sigma)_{X}\right), C(f(\widehat{X}))$ is polynomial in $\left.p\right\}$. The polynomial form of $C(f(\widehat{X}))$ assures locality of the counterterms and therefore the necessary condition for the renormalizability of the theory. On $\mathscr{D}_{H}$ the operator $C_{H}$ is defined by

$$
\left(C_{H} f\right)(\widehat{X})=\left\{\begin{array}{l}
f(\widehat{X}) \text { if either } X \cap H=\emptyset \text { or if } H \subset H^{\prime} \text { for some } H^{\prime} \in F_{X}, \\
C\left(f\left(\widehat{X}_{0}\right)\right) \text { if } X=H, \\
U_{H}\left(C\left(f\left(\widehat{H}_{0}\right)\right)\right) f(\widehat{X: H}) \text { if } H \notin F_{X} \text { and } F_{X} \cup\{H\} \in \mathscr{F}_{X}^{\prime}, \\
0 \text { else (overlapping diagrams), }
\end{array}\right.
$$

where $\widehat{X: H}=\left(X, F_{X} \cup\{H\},(\mathscr{C}, \sigma)_{X: H}\right)$ with $\left((\mathscr{C}, \sigma)_{H},(\mathscr{C}, \sigma)_{X: H}\right)$ being a pair of labelled forests which is in one-to-one correspondence with $(\mathscr{C}, \sigma)_{X}$, and $U_{H}$ is a transformation of momenta of the subdiagram $H$ of $X, U_{H}: \underline{q}_{H} \rightarrow \underline{q}_{H}-i e_{X H}^{T} \partial / \partial \underline{u}_{X}^{T}$, whose action on these momenta of the function $C\left(f\left(\widehat{H}_{0}\right)\right)$ describes the insertion of this function as a vertex into the reduced diagram(s) $X / F_{X} \cup\{H\}$. It is interesting to note that $\left(\left(1-C_{H}\right) f\right)(\widehat{X})$ gives a zero result for the first row in (2.14), $(1-C)\left(f\left(\widehat{X}_{0}\right)\right)$ if $X=H$ (second row), $f(\widehat{X})-U_{H}\left(C\left(f\left(\widehat{H}_{0}\right)\right)\right) f(\widehat{X: H})$ for the third row, and $f(\widehat{X})$ for the overlapping diagrams (fourth row). Nevertheless, when integrated over $\underline{\alpha}$ variables, 
the fourth row gives a zero result since for any two overlapping diagrams the variables of $\left(C_{H} f\right)(\widehat{X})$ of the first of overlapping diagrams and integration variables of the second overlapping diagram belong to different labelled forests, and according to property g. of labelled forests the integral is equal to zero. Also, it should be noted that the definition of the countertem (2.14) insures a correct Zimmermann forest formula, the validity of field equations and the action principle $[5,1]$.

2.7. Lemma 5: The expression for the counterterms includes the relation between the divergences of the subdiagram and corresponding divergence in diagram. This relation is assured by the Lemma 5 in [5]:

$$
U_{H}\left[\xi_{H}^{\omega_{H}} P\left(\underline{q}_{H}\right)\right] I_{G / H}=\left[\left(\omega_{H} !\right)^{-1} \frac{d^{\omega_{H}} I_{G}}{d t_{H}^{\omega_{H}}}\right]_{t_{H}=\underline{\tilde{u}}_{H}=0} .
$$

The Eq. (2.15) relates the singular terms of the $t_{H}$ integrals in amplitudes $\mathscr{T}_{G}$ and $\mathscr{T}_{H}$ where $H \subset G$, and $G / H$ is the corresponding reduced diagram whose amplitude is $\mathscr{T}_{G / H}$ (for generic $\mathscr{T}_{X}$ amplitude see (2.1)). The $I_{G}$ and $I_{G / H}$ are parts of the complete amplitudes $\mathscr{T}_{G}$ and $\mathscr{T}_{G / H}$ neglecting the integrations and operators the complete amplitudes have in common:

$$
\begin{aligned}
I_{G} & =Z_{H}\left(-i \frac{\partial}{\partial \tilde{\tilde{u}}_{H}}\right)\left(\operatorname{det} \tilde{M}_{G}\right)^{D / 2} \exp i W_{G}, \\
I_{G / H} & =\left(\operatorname{det} \tilde{M}_{G / H}\right)^{D / 2} \exp i W_{G / H},
\end{aligned}
$$

with $W_{G}$ and $W_{G / H}$ defined as in (2.1) for $G$ and $G / H$ and expressed in terms if of dimensionless momenta $\underline{\tilde{q}}_{X}$, auxiliary variables $\underline{\tilde{u}}_{X}$ and masses $\left(m_{X}^{2}=\sum_{\ell \in \mathscr{L}_{X}} \alpha_{\ell}\left(m_{\ell}-i \varepsilon\right)\right)$ with $X=G, G / H, H$. $P_{H}\left(\underline{q}_{H}\right)$ is an homogeneous polynomial of order $\omega_{H}$ appearing in the singular part of the $\mathscr{T}_{H}$ defined as in (2.11),

$$
\xi_{H}^{\omega_{H}} P\left(\underline{q}_{H}\right)=\left\{\frac{1}{\omega_{H} !} \frac{d^{\omega_{H}}}{d t_{H}^{\omega_{H}}}\left[Z\left(-i \frac{\partial}{\partial \underline{\tilde{u}}_{H}}\right)\left(\operatorname{det} \tilde{M}_{H}\right)^{D / 2} \exp i W_{H}\right]_{\underline{\tilde{\tilde{u}}}_{H}=0}\right\}_{t_{H}=0},
$$

obtained by integrating over $t_{H}$ in $\mathscr{T}_{H} . U_{H}$ is the same operator as in the definition of the counterterm. The singular part obtained by performing the $t_{H}$ integral of the $I_{G}$ amplitude is $\left[\left(\omega_{H} !\right)^{-1}\left(d / d t_{H}\right)^{\omega_{H}} I_{G}\right]_{t_{H}}=\tilde{\underline{u}}_{H} 0_{0}$. In the MS scheme the singular parts do not depend on $\mu$ [1].

2.8. $\mathbf{J}_{\mathbf{H}}^{\mathbf{K}}$ and $\tilde{\mathbf{J}}_{\mathbf{H}}^{\mathbf{K}}$ : In order to deal with the $t^{\nu h_{H}}$ factors in the amplitude (2.9) it is convenient to introduce two sets of functions $[5,1] J_{H}^{K}$ for $0 \leq K, h_{H}$, and $\tilde{J}_{H}^{K}$, for $0 \leq K \leq h_{H}$, defined for all $H \in \mathscr{C}, K$ being a nonnegative integer:

$$
\begin{aligned}
& J_{H}^{K}=\left\{f(\xi, v): f(\xi, v)=\xi^{v} \prod_{H^{\prime} \in \mathscr{M}(H)} g_{H^{\prime}}(\xi, v) \text { with } g_{H^{\prime}} \in \tilde{J}_{H}^{K_{H^{\prime}}}, K=\sum_{H^{\prime} \in \mathscr{M}(H)} K_{H^{\prime}}\right\} ; \\
& \tilde{J}_{H}^{K}=\left\{g(\xi, v): g \text { or } \xi^{v} g \text { with } g \in J_{H}^{K} \text { or } g(\xi, v)=\int_{1}^{\xi} \frac{d x}{x} f(x, v) \text { with } f \in J_{H}^{K-1}\right\} .
\end{aligned}
$$

The elements of sets $J_{H}^{K}$ and $\tilde{J}_{H}^{K}$ are defined iteratively by the equations (2.18). The initial function is defined for $h_{H}=0$, that is for $\mathscr{M}(H)=\emptyset$, implying $K=0$, for which there is only a set $J_{H}^{0}=\left\{\xi^{v}\right\}$ containing one function only, $\xi^{v}$. For a given $K$ the elements $f(\xi, v)$ of $J_{H}^{K}$ have the following properties [5]:

$$
\text { a. } f(\xi, v)=v^{-K} \sum_{m=1}^{h_{H}} c_{m} \xi^{v m} \text { with some constant } c_{m}
$$




$$
\begin{aligned}
& \text { b. } f(\xi, 0)=c(\ln \xi)^{K} \text { with some constant } c \text {; } \\
& \text { c. } f(\xi t, v)=\sum_{j} f_{1 j}(\xi, v) f_{2 j}(t, v) \text { with } f_{i j} \in J_{H}^{K_{i j}} \text { such that } K_{1 j}+K_{2 j}=K \\
& \text { d. } J_{H}^{0}=\xi^{m v}, m=1, \ldots, h_{H} .
\end{aligned}
$$

2.9. Proposition 3: The convergence of a labelled forest contribution to the renormalized amplitude $\mathscr{R}_{(\mathscr{C}, \sigma)}^{G}$, from Eq. (2.4), is assured by the proofs [5, 1] of Proposition 3 from Ref. [5]. The proof in Ref. [1] is done including the (regularization mass scale) factors $\mu^{v}$. The Proposition 3 states the following:

For any forest $\mathscr{X}_{0} \in \mathscr{F}_{G}^{\prime}$ satisfying condition $\mathscr{X}_{0} \subset \mathscr{C}$, one defines forests $\mathscr{X}_{H}$ each one satisfying a condition

$$
\mathscr{X}_{H}=\left\{H^{\prime} \in \mathscr{C}: H^{\prime} \subseteq H \text { for some } H \in \mathscr{X}_{0}\right\} .
$$

After performing all subtractions corresponding to subgraphs $H^{\prime} \in \mathscr{X}$, the contribution of $(\mathscr{C}, \sigma)$ to $\mathscr{R}_{G, \varepsilon}$ is a sum of terms of the form

$$
\prod_{H_{1} \in \mathscr{C} \backslash \mathscr{X}_{H}}\left\{\left.\int d \mu_{H_{1}}\left(1-C_{H_{1}}\right) \zeta_{H_{1}}^{v-\omega_{\bar{H}_{1}}} Z_{H_{1}}\left(-i \frac{\partial}{\partial \tilde{u}}\right)\left\{\prod_{H_{2} \in \mathscr{X}_{0}^{\prime}} \xi_{H_{2}}^{-\omega_{H_{2}}} g_{H}\left(\xi_{H_{2}}, v\right)\right\} g_{\mathscr{X}_{H}}(\underline{\tilde{q}}, \underline{\tilde{u}}, \underline{t}, \underline{\beta}, v)\right|_{\underline{\tilde{u}}=0}\right\} .
$$

Here $(\underline{t}, \beta)$ and $\tilde{u}$ are scaling variables and $\tilde{u}$-variables for $G / \mathscr{X}_{0}$ (in particular this means that $\underline{u}_{H}$ for $H \in \overline{\mathscr{X}}$ are already set to zero), $\tilde{q}$ are momenta for the family $\mathscr{C} / \mathscr{X}$, and $\mathscr{X}_{0}^{\prime}=\mathscr{X}_{0} \backslash(\mathscr{M}(H) \cup$ $\{H\})$. Furthermore, $g_{H}$ are elements of $\tilde{J}_{H}^{K}$ for some nonnegative integer $K$ and $g_{\mathscr{X}}$ is some element of the abstract algebra of covariants with coefficients which are $\mathbf{C}^{\infty}$ in scaling variables $(\underline{t}, \underline{\beta})$, analytic at $v=0$, and due to $\varepsilon>0$, exponentialy decreasing at $t_{G} \rightarrow \infty$.

The proof of Proposition 3 is based on the Lemma 5 and on the properties the functions of the sets $J_{H}^{K}$ and $\tilde{J}_{H}^{K}$.

The following should be noted:

a. The function $\left.g_{\mathscr{X}}(\underline{\tilde{q}}, \underline{\tilde{u}}, \underline{t}, \underline{\beta}, v)\right|_{\underline{\tilde{u}}=0}$ is an element of $G / H$ where $H \in \mathscr{X}_{0}$ which defines $\mathscr{X}_{H}$; $\emptyset \in \mathscr{X}_{0}$ so one might have $\bar{X}=\emptyset$.

b. In Eq. (2.21), due to the properties of labelled forests, only one term (this is related to the problem of decomposing integral region $\mathscr{D}(\mathscr{C}, \sigma)$ into Hepp sectors [25], defined as regions of Schwinger parameters satisfying relation $\alpha_{i_{1}} \leq \cdots \leq \alpha_{i_{j}} \leq \cdots \leq \alpha_{L}$, which are confirmed to be labelled forests in $[12,5])$ contributes per each $d \mu_{H_{1}}$ integration, so the complete expression for a given labelled $(\mathscr{C}, \sigma)$ forest has one term per integration,

$$
\begin{aligned}
& \left(\mathscr{R}_{v, \varepsilon}^{G}\right)_{(\mathscr{C}, \sigma)_{G}}=\prod_{H_{1} \in \mathscr{C} \backslash \mathscr{X}_{H}}\left\{\left(1-C_{H_{1}}\right) \int d \mu_{H_{1}} \zeta_{H_{1}}^{v-\omega_{H_{1}}}\left[\prod_{H_{2} \in \mathscr{M}^{\prime}\left(H_{1}\right)} \xi_{H_{2}}^{-\omega_{H_{2}}} g_{H_{2}}\left(\xi_{H_{2}}, v\right)\right]\right. \\
& \left.\times\left(Z_{H_{1}}\left(-i \frac{\partial}{\partial \tilde{u}}\right) g_{\mathscr{X}_{H}}(\underline{\tilde{q}}, \underline{\tilde{u}}, \underline{t}, \underline{\beta}, v)\right)_{\tilde{\underline{u}}_{H}=0}\right\} \\
& =\prod_{H_{1} \in \mathscr{C} \backslash \mathscr{X}_{H}}\left\{\left(1-C_{H_{1}}\right) \int d \mu_{H_{1}}\left[\zeta_{H_{1}}^{v-\omega_{H_{1}}} g_{H}\left(\xi_{H}, v\right)\left(Z_{H_{1}}\left(-i \frac{\partial}{\partial \tilde{u}}\right) g_{\mathscr{X}_{H}}(\underline{\tilde{q}}, \underline{\tilde{u}}, \underline{t}, \underline{\beta}, v)\right)_{\tilde{u}_{H}=0}\right]\right\},
\end{aligned}
$$

where we have used $\mathscr{M}^{\prime}\left(H_{1}\right)=\mathscr{M}\left(H_{1}\right) \backslash \mathscr{M}\left(H_{2}\right)=\{H\}$ and $\zeta_{H_{1}}^{v-\omega_{\bar{H}_{1}}} \xi_{H_{2}}^{-\omega_{H}}=\zeta_{H_{1}}^{v-\omega_{H_{1}}}$ and the definition of the counterterm. The choice of labelling $\sigma$ corresponds to the various choices of definition 
of variables $(\underline{t}, \underline{\beta})(2.5)$ for the maximal forest $\mathscr{C}$. These choices correspond to the ordering of the subsets in labelled forests introduced by Bonneau, $i<j$ if $t_{H_{i}}<t_{H_{j}}$ or $t_{H_{i}}=t_{H_{j}}$ and $\beta_{i}<\beta_{j}$ [1]. The steps of the algorithm given by Eq. (2.22), starting form the subtracted amplitude for a subdiagram $g_{H}$, give the subtracted amplitude $g_{H_{1}}$ (the expression in the curly brackets; it plays the role of $g_{H}$ in the next step of the algorithm, while the role of the subset $H_{1}$ is taken by the next in size $H_{1}$ subset in the sense of the Bonneau ordering) for the next largest subdiagram in the labelled forest $(\mathscr{C}, \sigma)$ in the sense of the Bonneau ordering. In the last step, after performing all steps except for the action of the $\left(1-C_{G}\right)$ operator, one obtains the relation between the completely subtracted diagram $\left(\mathscr{R}_{v, \varepsilon}^{G}\right)_{(\mathscr{C}, \sigma)_{G}}$ and the corresponding amplitude without the overall subtraction $\left(\overline{\mathscr{R}}_{v, \varepsilon}^{G}\right)_{(\mathscr{C}, \sigma)_{G}}$,

$$
\left(\mathscr{R}_{v, \varepsilon}^{G}\right)_{(\mathscr{C}, \sigma)_{G}}=\left(1-C_{G}\right)\left(\overline{\mathscr{R}}_{v, \varepsilon}^{G}\right)_{(\mathscr{C}, \sigma)_{G}},
$$

which is central for the Bonneau identities.

c. Prior to performing operations in Eq. (2.22), all the subtractions corresponding to subgraphs $H^{\prime} \in X, H^{\prime} \subseteq H$, have to be performed using the same algorithm as in (2.22), but for $H$ playing the role of $G$. These subtractions are making the $g_{\mathscr{X}}$, for subgraph $H$ functions, analytic, concerning the integrations over $(\underline{t}, \underline{\beta})_{H}$ variables corresponding to the subgraph $H$ and all its subsets $H^{\prime} \subset H$, with the property $H^{\prime} \in \mathscr{C}$. The procedure continues till one reaches an one loop subgraph. In a real calculation the procedure goes in the opposite way: one first performs the removal of divergences for smaller graphs using the $\left(1-C_{H}\right)$ and then proceeds to larger ones. This procedure can formally be written, following Bonneau, using the symbol $\bigcirc$, representing the application of the algorithm splitted into two parts [1],

$$
\begin{aligned}
\left(\mathscr{R}_{v, \varepsilon}^{G}\right)_{(\mathscr{C}, \sigma)_{G}} & =\left\{\prod_{H \in \mathscr{C} \backslash H_{i}} \int d \mu_{H}\left(1-C_{H}\right)\right\} I^{G / H_{i}} \bigcirc\left(\mathscr{R}_{v, \varepsilon}^{H_{i}}\right)_{\left(\mathscr{C}_{2}^{i}, \sigma_{2}\right)_{H_{i}}} \\
& =\left(\mathscr{R}_{v, \varepsilon}^{G / H_{i}}\right)_{\left(\mathscr{C}_{1}^{i}, \sigma_{1}\right)_{G / H_{i}}} \bigcirc\left(\mathscr{R}_{v, \varepsilon}^{H_{i}}\right)_{\left(\mathscr{C}_{2}^{i}, \sigma_{2}\right)_{H_{i}}},
\end{aligned}
$$

where $H_{1}$ is the first and $G=H_{h_{G}}\left(h_{G}\right.$ is number of loops of $G$ ) the last subgraph in the sense of Bonneau, and at the same time the overall graph $G$. It should be noted that the integrand $g_{\mathscr{X}}$ does not split into two parts as formally noted. Nevertheless, the powers of $\mu^{v}$ can be split into corresponding $G / H_{i}$ and $H_{i}$ factors.

d. Theorem of renormalizability: From Proposition 3 follows the theorem on renormalizability ([5]):

The singular part of the dimensionally regularized amplitude for any subgraph $H$ of a graph $G$ consists of poles of order $\leq h_{H}$, and is a polynomial of degree $\omega_{H}$ in the external momenta of the graph. The singular part vanishes if $H$ is superficially convergent. The amplitudes $\mathscr{R}_{G, \varepsilon}(\underline{p}, D)$, remaining after performing subtractions corresponding to all 1PI subgraphs, are analytic at $\bar{D}=4$ at any order of pertubation theory. The limit $D \rightarrow 4$ includes first the limit $v=4-D \rightarrow 0$ and then setting all evanescent objects to be equal to zero. The limit $\lim _{\varepsilon \rightarrow 0} \mathscr{R}_{G, \varepsilon}$ exists in the space of external momenta and is analytic at $D=4$, and it represents the renormalized amplitude $\mathscr{R}_{G}(\underline{p})$.

\section{Bonneau identities; Restauration of the BRST invariance in view of Bonneau identities}

The derivation of the Bonneau identities do have BMHV renormalization scheme background 
but are also based on two lemmas [1] which are presented in the following.

\subsection{Two Lemmas}

3.1.1. Lemma 1.: For any function $f(v)$ meromorphic in $v=4-D$, having poles at $v=0$, and whose Laurent expansion around $v=0$ is $f(v)=\sum_{-\infty}^{\infty} a_{i} v^{i}$, the following relations hold:

$$
\begin{aligned}
& f(v)=C(f(v))+(1-C)(f(v)), \\
& \text { r.s.p. }(f(v)) \equiv a_{-1}=v(C(f(v)))-C(v f(v)), \\
& \text { r.s.p. }(f(v))=(1-C)(v f(v))-v(1-C)(f(v)) .
\end{aligned}
$$

Here $C=p . p$. denotes the extraction of the singular part (principal part) of any function $f(v)$, $C(f(v))=\sum_{-\infty}^{-1} a_{i} v^{i}$; r.s.p. is the abbreviation for "residue of the simple pole", $a_{-1}$. The elements $A$ of the algebra $\mathscr{A}_{G}$, defined above Eq. (2.14) given in the precious section, are meromorphic functions of $v$ having poles at $v=0$. For the algebra $\mathscr{A}_{G}$ of any graph $G$, the operator $C$ defined in Eq. (2.13) plays the role of $C$ defined for general meromorphic function in $v, f(v)$ (here and in what follows the MS renormalization scheme is assumed).

3.1.2. Lemma 2.: The Lemma 2. relates the amplitudes of two Feynman graphs $G$ and $G^{v}$ which are identical up to one vertex $V$, being equal to $V$ in the first, and to $V^{v}=v V$ in the second amplitude:

$$
\left(\mathscr{R}_{v, \varepsilon}^{G^{v}}\right)_{(\mathscr{C}, \sigma)_{G}}-v\left(\mathscr{R}_{v, \varepsilon}^{G}\right)_{(\mathscr{C}, \sigma)_{G}}=\sum_{\gamma_{i}} U_{\gamma_{i}}\left(\text { r.s.p. }\left(\overline{\mathscr{R}}_{v, \varepsilon}^{\gamma_{i}}\right)_{\left(\mathscr{C}_{2}, \sigma_{2}\right)_{\gamma_{i}}}\right)\left(\mathscr{R}_{v, \varepsilon}^{G / \gamma_{i}}\right)_{\left(\mathscr{C}_{1}, \sigma_{1}\right)_{G / \gamma_{i}}}
$$

In Eq. (3.2) the sum is performed over all subgraphs $\gamma_{i} \subseteq G$ containing $V$ as one of its vertices. The amplitude $\mathscr{R}_{v, \varepsilon}^{\gamma_{i}}=\left(1-C_{\gamma_{i}}\right) \overline{\mathscr{R}}_{v, \varepsilon}^{\gamma_{i}}=\left(1-U_{\gamma_{i}} C\right) \overline{\mathscr{R}}_{v, \varepsilon}^{\gamma_{i}}$ corresponds to the subtracted amplitude $g_{\mathscr{X}_{H}}$ in the Eq. (2.22) with the identification $H:=\gamma_{i}$. The pair of labelled forests $\left(\left(\mathscr{C}_{2}, \sigma_{2}\right)_{\gamma_{i}},\left(\mathscr{C}_{1}, \sigma_{1}\right)_{G / \gamma_{i}}\right)$ is in one-to-one correspondence with the labelled forest $(\mathscr{C}, \sigma)_{G}$. To the vertex $V$ corresponds a field monomial $\mathscr{O}_{\delta}$.

In order to prove the Lemma 2., it is convenient to subdivide the maximal forest $\mathscr{C}$ of the graph $G$ into three subforests; $F_{B}=\left\{b_{k}, k=1, \ldots o\right\}, F_{A}=\left\{a_{j}, j=1, \ldots p\right\}$, and $F_{V}=\left\{v_{i}, i=1, \ldots, q\right\}$ with $F_{A} \subset F_{A V}, F_{V} \subseteq F_{A V}$, and with the property that the vertex $V$ is neither a vertex of $b_{k}$ nor of $a_{j}$ for every $b_{k} \in F_{B}$ and for every $a_{j} \in F_{A}$, while $V$ is a vertex of every $v_{i} \in F_{V}$. In the sense of the subtraction procedure $F_{A V}$ and $F_{B}$ are independent, while $F_{A}$ and $F_{V}$ are dependent. The maximal elements of $F_{B}$ and $F_{A V}$ may have only one common vertex or may be connected by one propagator. Further, the elements of $F_{V}$ are assumed to be strictly ordered: $\gamma_{1} \subset \gamma_{2} \subset \cdots \subset \gamma_{q}$. The subtraction procedure may be taken to be performed consecutively with respect to all the elements of the forests $F_{B}$ first, then of the forest $F_{A}$ and at the end of the forest $F_{V}$. With this ordering the proof proceeds in two steps. First, the procedure does not differentiate the amplitudes of $G^{v}$ and $G$ at the level of the elements of $F_{B}$ and $F_{A}$, since the corresponding subgraphs have the same vertices and propagators in both amplitudes. The amplitudes start to differ with the smallest element of $F_{V}, v_{1}$. Second, for each $v_{i}$ starting with $v_{1}$ and ending with $v_{q}=G$, the following relations hold: $\mathscr{R}_{i}^{\gamma_{i}^{v}}=\left(1-U_{\gamma_{i}} C\right) \overline{\mathscr{R}}^{\gamma_{i}^{v}, G \supset \gamma_{i}}=U_{\gamma_{i}}(1-C) \overline{\mathscr{R}}^{\gamma_{i}^{v}, \gamma_{i}}=v \mathscr{R}^{\gamma_{i}}+\sum_{j \leq i} U_{\gamma_{j}}$ (r.s.p. $\left.\left(\overline{\mathscr{R}}_{v, \varepsilon}^{\gamma_{j}}\right)\right) \mathscr{R}_{v, \varepsilon}^{\gamma_{i} / \gamma_{j}}$, with $U_{G}=1$ and $\mathscr{R}_{v, \varepsilon}^{\gamma_{i} / \gamma_{i}}=1$, and identifying $C_{\gamma_{i}}=U_{\gamma_{i}} C$. The subtracted amplitude $\overline{\mathscr{R}}^{\gamma_{i}^{\prime}, G \supset \gamma_{i}}$ is considered as a part of the amplitude for the graph $G$, and $\overline{\mathscr{R}}_{i}^{\gamma_{i}, \gamma_{i}}$ is self-standing amplitude for the graph $\gamma_{i}$ inserted 
by $U_{\gamma_{i}}$ into larger subgraphs. In the expression $U_{\gamma_{i}}(1-C) \overline{\mathscr{R}}^{\gamma_{i}^{v}, \gamma_{i}}$ the Lemma 5 is used. With this procedure the Lemma 2 is proved.

Two facts should be noted. First, the matrix elements $\overline{\mathscr{R}}_{v, \varepsilon}^{\gamma_{j}}$ are strongly restricted since they have to be divergent. Second, by summing the result of Eq. (3.2) over all labelled forests $(\mathscr{C}, \sigma)$ and using Eq. (2.4), one obtains the result relating the total finite amplitudes $\mathscr{R}_{v, \varepsilon}^{G^{v}}$ and $\mathscr{R}_{v, \varepsilon}^{G}$,

$$
\left(\mathscr{R}_{v, \varepsilon}^{G^{v}}\right)-v\left(\mathscr{R}_{v, \varepsilon}^{G}\right)=\sum_{(\mathscr{C}, \sigma)} \sum_{\gamma_{i} \in \mathscr{C}} U_{\gamma_{i}}\left(\text { r.s.p. }\left(\overline{\mathscr{R}}_{v, \varepsilon}^{\gamma_{i}}\right)_{\left(\mathscr{C}_{2}, \sigma_{2}\right)_{\gamma_{i}}}\right)\left(\mathscr{R}_{v, \varepsilon}^{G / \gamma_{i}}\right)_{\left(\mathscr{C}_{1}, \sigma_{1}\right)_{G / \gamma_{i}}} .
$$

3.2. Basic Bonneau identity: The Lemma 2. may be used to obtain the corresponding operatorial identity in several steps, which we give with more details than in [1].

a. First is to use the basic result of the renormalization theorem that the singular part of the amplitude for a graph $\gamma_{i}$, subtracted from the divergences of all its 1PI subgraphs $\overline{\mathscr{R}}_{v, \varepsilon}^{\gamma_{j}}$, is a polynomial in the masses and the external momenta of degree (or superficial divergence of $\gamma_{i}$ ) $\delta_{\gamma_{i}}=4-d_{\gamma_{i}}$ for 4-dimensional field theories, where $d_{\gamma_{i}}=\sum_{k=1}^{n_{i}} d_{\phi_{k}}$ is the sum of the canonical dimensions of all fields coresponding to the external lines of the subgraph $\gamma_{i}$. Therefore, $\overline{\mathscr{R}}_{v, \varepsilon}^{\gamma_{j}}$ can be written as the finite Taylor expansion in external momenta

$$
\text { r.s.p. } \overline{\mathscr{R}}_{v, \varepsilon}^{\gamma_{i}}=\sum_{r=0}^{\delta_{\gamma_{i}}} \sum_{\substack{\left\{i_{1}, \ldots, i_{r}\right\} \\ 1 \leq i_{j} \leq n \gamma_{i}}} \frac{1}{r !}\left[\frac{\partial^{r}}{\partial p_{i_{1}}^{\mu_{1}} \ldots \partial p_{i_{r}}^{\mu_{r}}} \text { r.s.p. } \overline{\mathscr{R}}_{v, \varepsilon}^{\gamma_{i}}\right]_{p_{i}=0} p_{i_{1}}^{\mu_{1}} \ldots p_{i_{r}}^{\mu_{r}},
$$

where $n_{\gamma_{i}}$ is the total number of external lines for the subgraph $\gamma_{i}$.

b. Inserting this result into (3.2), and rearranging the sum $\sum_{\gamma_{i}}$ over subgraphs $\gamma_{i}$, with respect to the number of external lines $n$, Eq. (3.2) becomes equal to

$$
\begin{aligned}
& \left(\delta \mathscr{R}_{v, \mathcal{E}}^{G^{v}, v G}\right)_{(\mathscr{C}, \sigma)_{G}} \equiv\left(\mathscr{R}_{v, \varepsilon}^{G^{v}}\right)_{(\mathscr{C}, \sigma)_{G}}-v\left(\mathscr{R}_{v, \varepsilon}^{G}\right)_{(\mathscr{C}, \sigma)_{G}}=\sum_{n=n_{\min }=2}^{n_{\max }=4} \sum_{\substack{\gamma_{i}^{n}: V \in \gamma_{i}^{n} \subseteq G \\
0 \leq \delta_{\gamma_{i}^{n}} \leq 4}} \sum_{r=0}^{\delta_{\gamma_{i}}} \sum_{\substack{\left\{i_{1}, \ldots, i_{r}\right\} \\
1 \leq i_{j} \leq n}} \\
& \left\{\left.\left(\frac{\partial^{r}}{\partial p_{i_{1}}^{\mu_{1}} \ldots \partial p_{i_{r}}^{\mu_{r}}} \text { r.s.p. } \overline{\mathscr{R}}_{v, \varepsilon}^{\gamma_{i}^{n}}\right)\right|_{p_{i}=0} U_{\gamma_{i}}\left(\frac{1}{r !} p_{i_{1}}^{\mu_{1}} \ldots p_{i_{r}}^{\mu_{r}}\right)\right\} \mathscr{R}_{v, \varepsilon}^{G / \gamma_{i}^{n}} \text {, }
\end{aligned}
$$

where $\gamma_{i}^{n}$ denotes $\gamma_{i}$ subgraph with $n$ external lines. Due to the fact $\gamma_{i}$ is 1 PI subgraph of $G, n_{\min }=2$. In 4-dimensional theories only terms with $n \leq 4$ may lead to divergences, therefore $n_{\max }=4$.

c. Since r.s.p. $\overline{\mathscr{R}}_{v, \varepsilon}^{\gamma_{i}^{n}}$ is a finite polynomial in external momenta, one can attribute to each term of the polynomial a local vertex of the normal product (see Ref. [26]) of the fields coresponding to the external lines of $\gamma_{i}$. The definition of the normal product of an operator inserted into some diagram, includes the renormalization of the operator and fields included in the diagram. The renormalization includes the subtraction, the limit $v, \varepsilon \rightarrow 0$ and setting all evanescent objects to zero. With the convention that all the momenta are incoming into graphs, the following replacement is valid

$$
R E P \equiv \frac{1}{r !} p_{i_{1}}^{\mu_{1}} \ldots p_{i_{r}}^{\mu_{r}} \rightarrow \frac{i^{r}}{r !} \frac{-i}{\prod_{j_{k}} n_{j_{k}} !} N\left[\prod_{k=n_{i}}^{1} \prod_{\alpha / i_{\alpha}=k} \partial_{\mu_{\alpha}} \phi_{j_{k}}(x)\right]
$$




$$
=\frac{i^{r}}{r !} \frac{-i}{n !} \sum_{\left\{j_{1}, \ldots, j_{n}\right\}} N\left[\prod_{k=n_{i}}^{1} \prod_{\alpha / i_{\alpha}=k} \partial_{\mu_{\alpha}} \phi_{j_{k}}(x)\right]
$$

where $\phi_{j_{k}}$ denotes a field with quantum numbers $j_{k}$ which define the type of the field. The identification (3.6) is obtained by taking a Feynman rule for its LHS. The factor $-i$ cancels the factor $i$ from $i \times$ Lagrangian, and the factor $1 / \prod_{j_{k}} n_{j_{k}}$ ! cancels the factor $\prod_{j_{k}} n_{j_{k}}$ ! coming from the identical fields on the RHS of the first line expression. In the second row we sum over all combinations of different types of the fields giving an additional factor $n ! / \prod_{j_{k}} n_{j_{k}}$ !, whose calculation gives the factor $1 / n$ !, $n$ being number of fields. According to the Lemma 5 of BM in the subsection 2.7 this vertex is inserted into $\mathscr{R}_{v, \varepsilon}^{G / \gamma_{i}^{n}}$. It should be noted that 1PI matrix element has a nontrivial group structure corresponding to the quantum numbers of the amputated legs $j_{k}$. Therefore, all the coefficients of the polynomial r.s.p. $\overline{\mathscr{R}}_{v, \varepsilon}^{\gamma_{i}^{n}}$ are group structures which do appear in the 1PI matrix element, multiplied by a certain power of masses, this power being smaller or equal to $\delta_{\gamma_{i}^{n}}$. In the case of a massless theory, the only contribution from the third sum in (3.5) comes from the term $r=\delta_{\gamma_{i}^{n}}$, and only dimensionless group structures do appear as coefficients. The choices of $\left\{i_{1}, \ldots, i_{r}\right\}$ indices in the fourth sum in Eq. (3.5) correspond to different choices of derivatives of $\phi_{j_{k}}$ fields. The total quantum numbers of the product of fields $\prod_{k=n}^{1} \phi_{j_{k}}(x)$ emerging from the diagram $\gamma_{i}$ are equal to the quantum numbers of the monomial $\mathscr{O}_{\delta}$ as a whole. The set of all such operators for all choices of subdiagrams $\gamma_{i}$ permitted by the quantum numbers of $\mathscr{O}_{\delta}$, and by the condition that the corresponding contributions to $\overline{\mathscr{R}}^{\gamma_{i}^{n}}$ are divergent, form a basis. In the limit $v, \varepsilon \rightarrow 0$, any matrix element $\left\langle 0\left|T\left(N\left[v \mathscr{O}_{\delta}\right]\right)\right| 0\right\rangle^{1 P I}$ may be expressed in terms of this basis, but one has to sum over all combinations of fields $\phi_{j_{1}} \ldots \phi_{j_{n}}$, that might contribute to the matrix element $\left\langle 0\left|T\left(N\left[v \mathscr{O}_{\delta}\right] X\right)\right| 0\right\rangle^{1 P I}$, with $X$ being an arbitrary polynomial product of the fields of the considered theory,

$$
\begin{aligned}
& \left\langle 0\left|T\left(N\left[v \mathscr{O}_{\delta}(x)\right] X\right)\right| 0\right\rangle^{1 P I}=\left.\sum_{(\mathscr{C}, \sigma)}\left(\boldsymbol{\delta} \mathscr{R}_{v, \varepsilon}^{G^{v}, v G}\right)_{(\mathscr{C}, \sigma)_{G}}\right|_{R E P} \\
& =\sum_{n=2}^{4} \sum_{J_{n}} \sum_{r=0}^{\delta_{O}^{J_{n}}} \sum_{\substack{\left\{i_{1}, \ldots, i_{r}\right\} \\
1 \leq i_{j} \leq n}}\left\{\frac{i^{r}}{r !} \frac{\partial^{r}}{\partial p_{i_{1}}^{\mu_{1}} \ldots \partial p_{i_{r}}^{\mu_{r}}} \text { r.s.p. }\left.\overline{\left\langle 0\left|T\left(N\left[\mathscr{O}_{\delta}(x)\right] \tilde{\phi}_{j_{1}}\left(p_{1}\right) \ldots \tilde{\phi}_{j_{n}}\left(p_{n}\right)\right)\right| 0\right\rangle}\right|_{p_{i}=0} ^{1 P I}\right\} \\
& \times\left\langle 0\left|T\left(N\left[\frac{-i}{n !} \prod_{k=n}^{1} \prod_{\alpha / i_{\alpha}=k} \partial_{\mu_{\alpha}} \phi_{j_{k}}(x)\right] X\right)\right| 0\right\rangle^{1 P I} .
\end{aligned}
$$

Here $J_{n}=\left\{j_{1}, \ldots, j_{n}\right\}$ is a set of different quantum numbers denoting the fields $\phi_{j_{k}}$. The fields $\tilde{\phi}_{j_{k}}$ are the Fourier-transformed fields $\phi_{j_{k}}$, and $\left.\langle 0| T\left(N\left[\mathscr{O}_{\delta}(x)\right] \tilde{\phi}_{j_{1}}\left(p_{1}\right) \ldots \tilde{\phi}_{j_{n}}\left(p_{n}\right)\right)\right]|0\rangle^{1 P I}=\mathscr{R}_{v, \varepsilon}^{\gamma_{i}}$.

d. Since (3.7) is valid for any set of fields $X$ it is valid at the operator level

$$
\begin{aligned}
& N\left[v \mathscr{O}_{\delta}(x)\right]=\sum_{n=2}^{4} \sum_{\left\{j_{1}, \ldots, j_{n}\right\}} \sum_{r=0}^{\delta_{0}^{J_{n}}} \sum_{\substack{\left\{i_{1}, \ldots, i_{r}\right\} \\
1 \leq i_{j} \leq n}}\left\{\frac{i^{r}}{r !} \frac{\partial^{r}}{\partial p_{i_{1}}^{\mu_{1}} \ldots \partial p_{i_{r}}^{\mu_{r}}}\right. \\
& \left.\quad \times \text { r.s.p. }\left.\left.\overline{\left.\langle 0| T\left(N\left[\mathscr{O}_{\delta}(x)\right] \tilde{\phi}_{j_{1}}\left(p_{1}\right) \ldots \tilde{\phi}_{j_{n}}\left(p_{n}\right)\right)\right]|0\rangle}\right|^{P I}\right|_{p_{i}=0}\right\} N\left[\frac{-i}{n !} \prod_{k=n}^{1} \prod_{\alpha / i_{\alpha}=k} \partial_{\mu_{\alpha}} \phi_{j_{k}}(x)\right],
\end{aligned}
$$

and this relation is the basic Bonneau identity. This equation confirms that the quantum numbers of the operator $O_{\delta}$ and of the product of derivarives of field operators in the last factor of the RHS 
of Eq. (3.8) are the same. The corresponding dimensions do not have to be the same, since the coefficients in the curly brackets may comprise masses, but in the case of a massless theory they are the same. Furthermore, the fields in the mentioned product may contain an external source field and in that case the combination of the Fourier-transformed fields must contain a product of the Fourier-transformed fields, which in combination with the external source field form a term in the action of the considered model. The external source field may be put outside of the normal pruduct $N$, but the derivatives acting on it have to be retained within it.

$X$ is any set of fields which arise from the action of the theory and form together with $v \mathscr{O}_{\delta}(x)$ a 1PI diagram, with $X$ fields being external fields of the 1PI diagram. Therefore, the relation (3.7) may be written for the sum of such fields or, in other words, for the sum of matrix elements $\left\langle 0\left|T\left(N\left[\mathscr{O}_{\delta}(x)\right] X\right)\right| 0\right\rangle$ which are represented in the field theory by the insertion of the $v \mathscr{O}_{\delta}(x)$ operator into the renormalized effective action $\Gamma_{r e n},\left[\nu \mathscr{O}_{\delta}(x)\right] \cdot \Gamma_{r e n}$. In the same way one can represent the right hand side of Eq. (3.7). This leads to the equality

$$
\begin{aligned}
& N\left[v \mathscr{O}_{\delta}(x)\right] \cdot \Gamma_{r e n}=\sum_{n=2}^{4} \sum_{J_{n}} \sum_{\substack{r=0 \\
\delta_{0}^{J_{n}}}}^{\substack{\left.j_{1}, \ldots, i_{r}\right\} \\
1 \leq i_{j} \leq n}}\left\{\frac{i^{r}}{r !} \frac{\partial^{r}}{\partial p_{i_{1}}^{\mu_{1}} \ldots \partial p_{i_{r}}^{\mu_{r}}}\right. \\
& \left.\times \text { r.s.p. }\left.\left.\overline{\left\langle 0\left|T\left(N\left[\mathscr{O}_{\delta}(x)\right] \tilde{\phi}_{j_{1}}\left(p_{1}\right) \ldots \tilde{\phi}_{j_{n}}\left(p_{n}\right)\right)\right| 0\right\rangle}\right|^{P I}\right|_{p_{i}=0}\right\} N\left[\frac{-i}{n !} \prod_{k=n}^{1} \prod_{\alpha / i_{\alpha}=k} \partial_{\mu_{\alpha}} \phi_{j_{k}}(x)\right] \cdot \Gamma_{r e n} .
\end{aligned}
$$

\subsection{Bonneau identity for the trace anomaly}

3.3.1. Trace anomaly and axial anomaly: The properties of the normal product given in subsection 3.2. point c. imply that the evanescent metric $\widehat{g}^{\mu \nu}$ does not commute with the normal product, in contract to 4 -dimensional metric $\bar{g}^{\mu v}$, which is not affected by renormalization procedure and does commute with the normal product. The information on the evanescent object may be received only if, in the procedure of taking normal form [5] of the amplitude, $\widehat{g}_{\mu}^{\mu}=-v=-(4-D)$ appear and divergences appearing in the integration procedure multiply $v$ and give an additional finite contribution to the amplitude. This implies the following trace anomaly relations [2]

$$
\begin{aligned}
& g^{\mu v} N\left[g_{\mu v} P(\phi, \partial \phi)\right]-N\left[g_{\mu}^{\mu} P(\phi, \partial \phi)\right]=N[v P(\phi, \partial \phi)] \\
& g^{\mu v} N\left[\mathscr{O}_{\mu v \lambda \ldots}(\phi, \partial \phi)(x)\right]-N\left[g^{\mu v} \mathscr{O}_{\mu v \lambda \ldots}(\phi, \partial \phi)(x)\right]=N\left[-\widehat{g}^{\mu v} \mathscr{O}_{\mu v \lambda \ldots}(\phi, \partial \phi)(x)\right]
\end{aligned}
$$

where $P(\phi, \partial \phi)(x)$ and $\mathscr{O}_{\mu \nu \lambda \ldots}(\phi, \partial \phi \ldots)(x)$ are scalar and tensor monomials in the fields and their derivatives. The operatorial relations written above have to be understood as the insertion of the $N[\ldots]$ operators as a vertex into an arbitrary 1PI diagram, labeled with its external fields designated by $X$, and taking its vaccum expectation value, $N[\ldots] \rightarrow\langle 0|T(N[\ldots] X)| 0\rangle$.

The axial anomaly is a consequence of the fact that in the BMHV scheme, $\gamma_{5}$ does not anticommute with the $D$-dimensional $\gamma^{\mu}$ matrices: it anticommutes with the 4-dimensional $\bar{\gamma}^{\mu}$ matrices, but commutes with the $(-v)$-dimensional $\widehat{\gamma}^{\mu}$ matrices, leading to the equality $\left\{\gamma_{\mu}, \gamma_{5}\right\}=2 \widehat{\gamma}_{\mu} \gamma_{5}$. The tree level term $2 \widehat{\gamma}_{\mu} \gamma_{5}$ induces at the loop level additional contribution to the nonconservation of the axial current [2], called axial anomaly. The evanescent tree level term inducing the axial anomaly may have different forms [6, 27], depending on how the fermion-fermion-gauge boson 
interaction is defined in $D$ dimensions, all of which are equivalent at 4 dimensions. For instance, if the mentioned interaction term has the form $\bar{\psi} P_{L} \gamma_{\mu} P_{R} \psi A^{\mu}$, the evanescent term inducing the axial anomaly is $N\left[\widehat{g}^{\mu v} \bar{\psi} \gamma_{5} \gamma_{v}\left(1 / 2 \overleftrightarrow{\partial}_{\mu}\right) \psi\right]$ having the trace anomaly form (3.11). If the interaction term $\bar{\psi} \gamma_{\mu} P_{R} \psi A^{\mu}$ is chosen, then the evanescent term inducing axial anomaly contains the gauge field $N\left[\widehat{g}^{\mu v} \bar{\psi} \gamma_{5} \gamma_{v}\left(1 / 2 \overleftrightarrow{\partial}_{\mu}-i g A_{\mu}\right) \psi\right]$, which is again of the trace anomaly form (3.11).

In fact all spurious anomalies (those which are not proportional to the Levi-Civita symbol) have always the trace anomaly form. Therefore, it is enough the consider the Bonneau identities for the general trace anomaly (3.11).

3.3.2. Bonneau identities for the trace anomaly: The general form of the regularized Feynman integrand of any graph $H$, with external momenta $p_{i}$, and having a special vertex $V\left(\mathscr{O}_{\mu \nu \Lambda}\right) \equiv$ $V\left(\mathscr{O}_{\mu \nu \lambda_{1} \ldots}\right)$ corresponding to the field monomial $\mathscr{O}_{\mu \nu \Lambda} \equiv \mathscr{O}_{\mu \nu \lambda_{1} \ldots}$ with Lorentz indices $\mu, \nu$ to be contracted with $-\hat{g}^{\mu \nu}$ and remaining Lorentz indices $\Lambda=\lambda_{1}, \ldots$, (which may but no not have to be present in the special vertex) is

$$
I_{v, \varepsilon ; \mu v}^{H^{\theta_{\mu \nu \Lambda}}}=\sum_{i, j \in V_{H}} p_{\mu}^{i} p_{v}^{j} M_{1 i j}^{H^{\theta_{\mu v \Lambda}}}+g_{\mu v} M_{2}^{H^{\theta_{\mu \nu \Lambda}}}+\sum_{i \in V_{H}} p_{\mu}^{i} M_{3 v}^{H^{\theta_{\mu \nu \Lambda}}}+M_{4 \mu \nu}^{H^{\theta_{\mu \nu \Lambda}}}
$$

where the component functions $M_{1 i j}^{H^{\sigma_{\mu v \Lambda}}}$ and $M_{2}^{H^{\sigma_{\mu v \Lambda}}}$ do not have Lorentz indices $\mu$ and $v, M_{3 v}^{H^{\sigma_{\mu v \Lambda}}}$ has the Lorentz index $v$ and $M_{4 \mu v}^{H_{\mu \nu \Lambda}}$ is a tensor function antisymmetric in Lorentz indices $\mu$ and $v$. The integrand and the component functions may have any number of additional Lorentz indices $\Sigma=\sigma_{1}, \ldots$ independent of Lorentz indices $\Lambda$, but the results obtained are the same as those obtained below. The component functions may depend on the (remaining) external momenta the Levi-Civita symbols and $\gamma^{\alpha}$ matrices. Furthermore, $V_{H}$ is the set of vertices of graph $H$. The corresponding Feynman integrand of the same graph but with the evanescent operator $\Delta_{\Lambda} \equiv-\widehat{g}^{\mu \nu} \mathscr{O}_{\mu v \Lambda}$ is

$$
I_{v, \varepsilon}^{H^{\Delta_{\Lambda}}}=-\sum_{i, j \in V_{H}} \widehat{p_{i} \cdot p_{j}} M_{1 i j}^{H^{\sigma_{\mu \nu \Lambda}}}+v M_{2}^{H^{\theta_{\mu \nu \Lambda}}}-\widehat{p}_{i} \cdot \widehat{M_{3 i}^{H^{\sigma_{\mu \nu \Lambda}}}} .
$$

The results (3.12) and (3.13) may be used to find the difference between the regularized subtracted amplitudes $\mathscr{R}_{v, \mathcal{E}}^{G_{\Lambda}^{\Lambda_{\Lambda}}}$ and $\left(-\widehat{g}^{\mu v}\right) \mathscr{R}_{v, \mathcal{\varepsilon}}^{G^{\sigma} \mu v \Lambda}$, which represents the trace anomaly for the special vertex $V\left(\mathscr{O}_{\mu v \Lambda}\right)$. Using the same subdivision of the maximal forest $\mathscr{C}$ of considered labelled forest $(\mathscr{C}, \sigma)$ into subforests $F_{B}, F_{A}$ and $F_{V}$ as in the proof of the Bonneau's Lemma 2 from subsubsection 2.1.2, and performing the algorithm procedure of Eq. (2.22), one obtains the same amplitudes for all subgraphs $b_{k} \in F_{B}$ and $a_{j} \in F_{A}$ of $\mathscr{R}_{v, \varepsilon}^{G_{\Lambda}^{\Delta}}$ and $-\widehat{g}^{\mu v} \mathscr{R}_{v, \varepsilon}^{G^{\mathscr{O}_{\nu \nu \Lambda}}}$, since they do not contain the vertex $\mathscr{O}_{\mu v \Lambda}$. The differences do appear for all amplitudes of the subgraphs $v_{i} \in F_{V}, i=1, \ldots, q$, as explained in what follows. The integrand functions of $\int d \mu_{H_{i}}$ for graphs $v_{i}^{\Delta_{\Lambda}}$ and $v_{i}^{\mathscr{O}_{\mu v \Lambda}}$ have the same form as the expressions in (3.12) and (3.13), with the identification $H:=v_{i-1}$ and $H_{1}=v_{i}$ in (2.22),

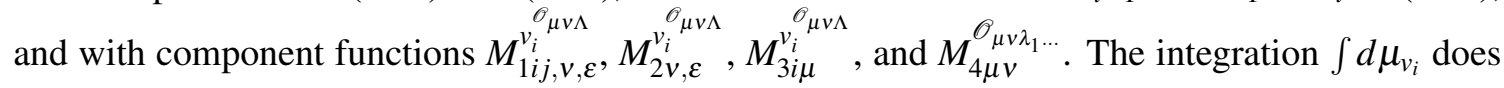
not change the Lorentz structure and the dependence on the external momenta, but it leads to the integrated component functions denoted by $\bar{M}_{1 i j, v, \varepsilon}^{v_{i}^{\sigma_{\mu v \Lambda}}}$ and $\bar{M}_{2 v, \varepsilon}^{v_{i}{ }^{\sigma_{\mu v \Lambda}}}, \bar{M}_{3 i \mu}^{v_{i}^{\sigma_{\mu v \Lambda}}}$ and $\bar{M}_{4 \mu \nu}^{\mathscr{O}_{\mu \nu \Lambda}}$, which have poles in $v$, and which are forming total amplitude without overall subtraction,

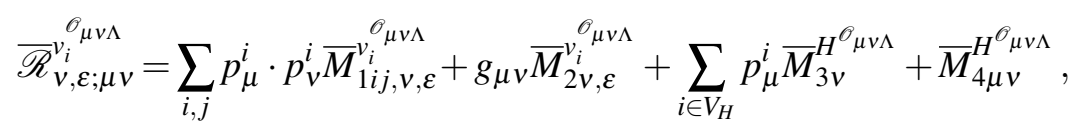




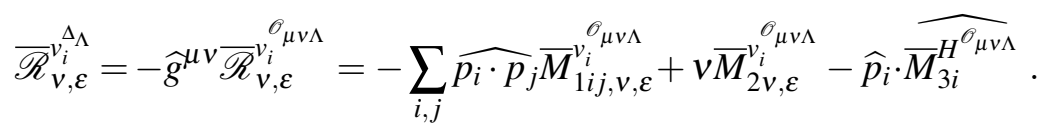

The action of the $U_{v_{i}}(1-C)$ operator on the amplitudes $\overline{\mathscr{R}}_{v, \varepsilon}^{v_{i}{ }^{\mu}{ }^{\mu \Lambda}}$ and $\overline{\mathscr{R}}_{v, \varepsilon}^{v_{i} \Delta_{\Lambda}}$ induces an additonal (polynomial) term $U_{v_{i}}\left(\operatorname{r.s.p}\left(\bar{M}_{v, \varepsilon}^{v_{i}^{O_{\mu v \Lambda}}}\right)\right)$ for the amplitude of the diagram $v_{i}^{\Delta_{\Lambda}}$ with respect to the $v_{i}^{\mathscr{O}_{\mu v \Lambda}}$ amplitude if it was multiplied by $-\widehat{g}^{\mu v}$. The mutiplication with $-\widehat{g}^{\mu v}$ serves only for comparison, since the real multiplication with $-\widehat{g}^{\mu v}$ is performed only after all steps of the algorithm, that is with the final amplitude $\mathscr{R}_{\mu v}^{G^{\mathcal{O}}{ }{ }}$. In addition, all the subsets $v_{j} \subset v_{i}$ lead, according to the BM Lemma 5 of subsection 2.7., to the terms of the form $U_{v_{j}}\left(\operatorname{r.s.p}\left(M_{v, \varepsilon}^{v_{j}^{{ }}{ }^{\mu \nu \Lambda}}\right)\right) \mathscr{R}^{v_{i} / v_{j}}$. At the next loop level, that is for $v_{i+1}$, the first and third term in the expression for $\overline{\mathscr{R}}_{v, \varepsilon}^{v_{i}}$ in Eq. (3.14) may constitute a source of new $v M_{v, \varepsilon}^{H^{\mathscr{C}} \mu v \Lambda}$ terms. At the level of the graph $G$, the final result is

$$
\left(\mathscr{R}_{v, \varepsilon}^{G^{\Delta \Lambda}}\right)_{(C, \sigma)}-\left(-\widehat{g}^{\mu v}\right)\left(\mathscr{R}_{v, \varepsilon ; \mu v}^{G^{\mathscr{O} \mu v \Lambda}}\right)_{(C, \sigma)}=\sum_{\substack{v_{i} \in \mathscr{C} \\ V \in v_{i}}} U_{v_{i}}\left(\text { r.s.p. }\left(\bar{M}_{v, \varepsilon}^{v_{i}{ }^{\mu}{ }^{\mu \nu \Lambda}}\right)\right)_{\left(\mathscr{C}_{2}, \sigma_{2}\right)_{v_{i}}}\left(\mathscr{R}_{v, \varepsilon}^{G / v_{i}}\right)_{\left(\mathscr{C}_{1}, \sigma_{1}\right)_{G / v_{i}}} \text {. }
$$

Summing this result over the labelled forest, the following result is obtained [2]

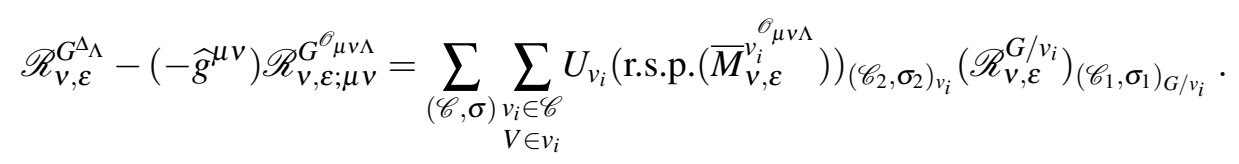

The only difference with respect to the result (3.3) is that this result (3.15) has a r.s.p. of the part of the subtracted amplitude $\bar{M}_{v, \varepsilon}^{v_{i}{ }_{\mu v \Lambda}}$, and not of the whole amplitude $\overline{\mathscr{R}}_{v, \varepsilon}^{v_{i}{ }_{\mu v \Lambda}}$. To achieve the same form as in Eq. (3.3), following Bonneau [2], a new symmetric tensor $\check{g}_{\mu v}$ is introduced, whose trace and contraction properties are:

$$
\breve{g}_{\mu}^{\mu}=1, \quad \breve{g}_{\mu v} g_{\rho}^{\nu}=\breve{g}_{\mu v} \widehat{g}_{\rho}^{v}=\breve{g}_{\mu \rho} .
$$

The amplitude $\overline{\mathscr{R}}_{v, \varepsilon}^{v_{i}{ }^{\mu}{ }^{\mu \nu \Lambda}}$ from Eqs. (3.14) and (3.16) is then contacted with $\check{g}^{\mu v}$ and fully simplified. The remaining terms with $\breve{g}_{\mu \nu}$ are then set to zero, and the r.s.p. of the final expression is taken:

$$
\begin{aligned}
& \text { r.s.p. }\left.\overline{\mathscr{R}}_{v, \varepsilon}^{v_{i}^{\check{\Delta}_{\Lambda}}}\right|_{\check{g}=0} \equiv \text { r.s.p. }\left.\left(\check{g}^{\mu v} \overline{\mathscr{R}}_{v, \varepsilon}^{v_{i}{ }^{O_{\nu \nu \Lambda}}}\right)\right|_{\check{g}=0}=\text { r.s.p. }\left(\sum_{i, j \in V_{v_{i}}}{\overline{p_{i} \cdot p_{j}}}_{\bar{M}_{1 i j, v, \varepsilon}^{v_{i}}}^{\sigma_{\mu v \Lambda}}+\bar{M}_{2 v, \varepsilon}^{v_{i} \mu v \Lambda}\right. \\
& \left.+\sum_{i \in V_{H}} \check{p}_{i} \cdot \bar{M}_{3 v, \varepsilon}^{v_{i}^{o_{\mu \nu \Lambda}}}\right)\left.\right|_{\check{g}=0}=\text { r.s.p.p. } \bar{M}_{1 v, \varepsilon}^{v_{i}^{O} \mu v}
\end{aligned}
$$

The insertion of this relation into (3.16) provides the explicit result,

$$
\mathscr{R}_{v, \varepsilon}^{G^{\Delta_{\Lambda}}}-\left(-\widehat{g}^{\mu v}\right) \mathscr{R}_{v, \varepsilon ; \mu v}^{G^{\mathscr{O}_{\mu \nu \Lambda}}}=\sum_{\gamma_{i}, V \in \gamma_{i}} U_{\gamma_{i}}\left(\text { r.s.p.p. }\left.\overline{\mathscr{R}}_{v, \varepsilon}^{\widetilde{\gamma_{\Lambda}}}\right|_{\breve{g}=0}\right) \mathscr{R}_{v, \varepsilon}^{G / \gamma_{i}} .
$$

It should be noted that in the loop integration procedure, new evanescent operators different from the monomial $\Delta_{\Lambda}$ might be induced, but all of these operators do contain one and only one $\left(-\widehat{g}^{\mu v}\right)$ 
from $\Delta_{\Lambda}$, which is singled out by replacing $\left(-\widehat{g}^{\mu v}\right)$ by $\breve{g}^{\mu \nu}$. Therefore, all induced evanescent terms depend linearly on the initial evanescent $\hat{g}^{\mu v}$ present in the evanescent vertex operator $\Delta_{\Lambda}$.

Performing the same procedure as for basic Bonneau identities given there by points a.,b.,c.,d. of subsection 3.2., one obtains

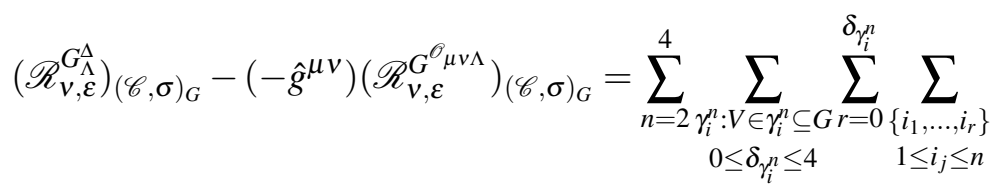

$$
\begin{aligned}
& \left\{\left.\left(\frac{\partial^{r}}{\partial p_{i_{1}}^{\mu_{1}} \ldots \partial p_{i_{r}}^{\mu_{r}}} \text { r.s.p. } \overline{\mathscr{R}}_{v, \varepsilon}^{\gamma_{i}^{n, \check{L}_{\Lambda}}}\right)\right|_{p_{i}=0} U_{\gamma_{i}}\left(\frac{1}{r !} p_{i_{1}}^{\mu_{1}} \ldots p_{i_{r}}^{\mu_{r}}\right)\right\} \mathscr{R}_{v, \varepsilon}^{G / \gamma_{i}^{n}}
\end{aligned}
$$

after the procedure steps of the points $\mathbf{a}$. and $\mathbf{b}$., then the result

$$
\begin{aligned}
& \left\langle 0\left|N\left[-\widehat{g}^{\mu v} \mathscr{O}_{\mu v \Lambda}(x)\right]\right| 0\right\rangle^{1 P I}=\sum_{n=2}^{4} \sum_{J_{n}} \sum_{r=0}^{\delta_{O}^{J_{n}}} \sum_{\substack{\left\{i_{1}, \ldots, i_{r}\right\} \\
1 \leq i_{j} \leq n}}\left\{\frac{i^{r}}{r !} \frac{\partial^{r}}{\partial p_{i_{1}}^{\mu_{1}} \ldots \partial p_{i_{r}}^{\mu_{r}}}\right. \\
& \left.\times \text { r.s.p. }\left.\left.\overline{\left\langle 0\left|T\left(N\left[\check{g}^{\mu v} \mathscr{O}_{\mu v \Lambda}(x)\right] \tilde{\phi}_{j_{1}}\left(p_{1}\right) \ldots \tilde{\phi}_{j_{n}}\left(p_{n}\right)\right)\right| 0\right\rangle}\right|^{P I}\right|_{\substack{p_{i}=0 \\
\grave{g}=0}}\right\} \\
& \times\left\langle 0\left|N\left[\frac{-i}{n !} \prod_{k=n}^{1}\left[\left(\prod_{\alpha / i_{\alpha}=k} \partial_{\mu_{\alpha}} \phi_{j_{k}}(x)\right)\right]\right]\right| 0\right\rangle^{1 P I},
\end{aligned}
$$

after the procedure steps of the point c. From the relation (3.21) one obtains

$$
\begin{aligned}
& N\left[-\widehat{g}^{\mu v} \mathscr{O}_{\mu v \Lambda}(x)\right] \cdot \Gamma_{r e n}=\sum_{n=2}^{4} \sum_{\left\{j_{1}, \ldots, j_{n}\right\}} \sum_{\substack { r=0 \\
\begin{subarray}{c}{\left\{i_{1}, \ldots ., i_{r}\right\} \\
1 \leq i_{j} \leq n{ r = 0 \\
\begin{subarray} { c } { \{ i _ { 1 } , \ldots . , i _ { r } \} \\
1 \leq i _ { j } \leq n } }\end{subarray}}^{\delta_{J_{n}}}\left\{\frac{i^{r}}{r !} \frac{\partial^{r}}{\partial p_{i_{1}}^{\mu_{1}} \ldots \partial p_{i_{r}}^{\mu_{r}}}\right. \\
& \left.\times \text { r.s.p. }\left.\overline{\left\langle 0\left|T\left(N\left[\widetilde{g}^{\mu v} \mathscr{O}_{\mu v \Lambda}(x)\right] \tilde{\phi}_{j_{1}}\left(p_{1}\right) \ldots \tilde{\phi}_{j_{n}}\left(p_{n}\right)\right)\right| 0\right\rangle}\right|_{\substack{p_{i}=0 \\
\grave{g}=0}}\right\} \\
& \times N\left[\frac{-i}{n !} \prod_{k=n}^{1} \prod_{\alpha / i_{\alpha}=k} \partial_{\mu_{\alpha}} \phi_{j_{k}}(x)\right] \cdot \Gamma_{r e n},
\end{aligned}
$$

by collecting all 1PI amplitudes with different choices of external fields $X$ into the effecive action. Finally, after the procedure steps of the point $\mathbf{d}$. one obtains the result

$$
\begin{aligned}
& N\left[-\widehat{g}^{\mu v} \mathscr{O}_{\mu v \Lambda}(x)\right]=\sum_{n=2}^{4} \sum_{\left\{j_{1}, \ldots, j_{n}\right\}} \sum_{r=0}^{\delta_{O}^{J_{n}}} \sum_{\left\{i_{1}, \ldots, i_{r}\right\}}\left\{\frac{i^{r}}{r !} \frac{\partial^{r}}{\partial p_{i_{1}}^{\mu_{1}} \ldots \partial p_{i_{r}}^{\mu_{r}}}\right. \\
& \left.\times \text { r.s.p. }\left.\left.\overline{\left\langle 0\left|T\left(N\left[\check{g}^{\mu v} \mathscr{O}_{\mu v \Lambda}(x)\right] \tilde{\phi}_{j_{1}}\left(p_{1}\right) \ldots \tilde{\phi}_{j_{n}}\left(p_{n}\right)\right)\right| 0\right\rangle}\right|^{P I}\right|_{\substack{p_{i}=0 \\
\grave{g}=0}}\right\} \\
& \times N\left[\frac{1}{n !} \prod_{k=n i_{\alpha}=k}^{1} \prod_{\mu_{\alpha}} \phi_{j_{k}}(x)\right] .
\end{aligned}
$$


The relation (3.23) is the Bonneau identity for the special vertex $V$ corresponding to the generic monomial $\mathscr{O}_{\mu \nu \Lambda}$. In the same way as in the basic Bonneau identity Eq. (3.8), the fields in the last term RHS of (3.23) may have an external source field per monomial, in which case the coefficient term must contain a product of Fourier-transformed fields, which together with the external source field form a term in the action of the considered theory. The external source field may be put outside the normal product $N$, but the derivatives acting on it have to be retained within $N$.

\subsection{BRST invariance in view of the Bonneau identities}

This subsection discusses two related topics. In the first subsubsection we review the regularized action principle, which constitutes a basis for the second one. The second subsubsection relates the breaking of the BRST symmetry to the operators induced by the tree-level evanescent operators, showing that the BRST breaking terms may be expressed using the Bonneau identities.

3.4.1. Regularized action principle. The regularized action principle states the following three equations hold in dimensionally regularized theories:

i. Arbitrary polynomial variations of the quantized fields $\phi, \delta \phi(x)=\delta \theta(x) P(\phi(x))$ leave invariant the dimensionally regularized the generating functional for the Green functions $Z_{D R e g}[4,5,6]$

$$
\delta Z_{D R e g}[J, K] \equiv\left\langle\delta\left(S_{f}+S_{I}\right) \exp \left\{\frac{i}{\hbar} S_{I}[\phi, J, K, \lambda]\right\}\right\rangle=0,
$$

where $\phi, \lambda, J$ and $K$ represent fields, couplings, sources for the fields and sources for their BRST tranformations, where $S_{f}$ is the free action defining propagators of the theory, $S_{I}=S_{I}[\phi, J, K, \lambda]=$ $S_{i}[\phi, K, \lambda]+\int d^{D} x J_{i}(x) \phi_{i}(x)$ is the interaction part of the action including the sources $J_{i}$ for all the fields $\phi_{i}=(\varphi, \Phi)_{i}, S_{i}[\phi, K, \lambda]=S_{0 i}[\phi, K, \lambda]+S_{s c t}^{(n)}[\phi, K, \lambda]+S_{f c t}^{(n)}[\phi, K, \lambda]$ is the interaction part of the action which includes the BRST sources $K_{\Phi_{i}}$ of the fields $\Phi_{i}$ which transform nonlinarly on BRST transformations, $S_{0 i}$ is the interaction part of the tree level action $S_{0}=S_{0 i}+S_{f}$, and $S_{s c t}^{(n)}$ and $S_{f c t}^{(n)}$ are singular and finite parts of the counterterm part of the counterterm action $S_{c t}^{(n)}=S_{s c t}^{(n)}+S_{f c t}^{(n)}$ including counterterms up to $n$-loop level. Finally, $Z_{D R e g}[J, K, \lambda]=\int \mathscr{D} \phi \exp \left\{i\left(S_{D R e g}^{(n)}+\int J_{i} \phi_{i}\right)\right\}$, and $\langle(\ldots)\rangle=\int \mathscr{D} \phi \exp \left\{i S_{f}\right\}(\ldots)$.

ii. Variations of external fields $E(x) \equiv(K(x), J(x))$ lead to equality $[3,5,6]$

$$
\left\langle\frac{\delta S_{I}}{\delta E(x)} \exp \left\{S_{I}[\phi, J, K, \lambda]\right\}\right\rangle=-i \hbar \frac{\delta Z_{D R e g}[J, K, \lambda]}{\delta E(x)} .
$$

iii. Variation of parameters give $[3,5,6]$

$$
\left\langle\frac{\delta\left(S_{f}+S_{I}\right)}{\delta \lambda} \exp \left\{S_{I}[\phi, J, K, \lambda]\right\}\right\rangle=-i \hbar \frac{\delta Z_{D R e g}[J, K, \lambda]}{\delta \lambda} .
$$

The corresponding regularized quantum action equations for the dimensionally regularized effective action $\Gamma_{D R e g}$ may be written. The equations (3.24-3.26) for the renormalized action which state the (renormalized) quantum action principle are defined as follows. First, the renormalized action is defined by taking the limit $\operatorname{LIM}_{v \rightarrow 0}$, denoting the limit when setting $v, \varepsilon \rightarrow 0$ and setting all evanescent objects to be equal to zero:

$$
\Gamma_{r e n}=\mathrm{LIM}_{v \rightarrow 0} \Gamma_{D R e g} .
$$


The same limit is applied to the regularized quantum action principle equations for the regularized effective action $\Gamma_{D R e g}$ to obtain the corresponding quantum action principle equations for the renormalized effective action $\Gamma_{\text {ren }}$.

3.4.2. Slavnov-Taylor identities and anomalous insertions: It is convenient to derive the SlavnovTaylor identities of the theory in a similar manner. The reason for this is that in the BMHV scheme the action of the D-dimensional Slavnov-Taylor opearator $\mathscr{S}_{D}$ on regularized effective action the terms coming from evanescent operators only [6],

$$
\begin{aligned}
& \mathscr{S}_{D}\left(\Gamma_{D R e g}\right) \equiv \int d^{D} x\left(s_{D} \phi\right) \frac{\delta \Gamma_{D R e g}}{\delta \phi}+\frac{\delta \Gamma_{D R e g}}{\delta K_{\Phi}} \frac{\delta \Gamma_{D R e g}}{\delta \Phi} \\
& =\Delta \cdot \Gamma_{D R e g}+\Delta_{c t} \cdot \Gamma_{D R e g}+\int d^{D} x\left[\frac{\delta S_{c t}^{(n)}}{\delta K_{\Phi}(x)} \cdot \Gamma_{D R e g}\right] \frac{\delta \Gamma_{D R e g}}{\delta \Phi(x)},
\end{aligned}
$$

where $s_{D}$ is BRST operator in $D$ dimensions. The relation (3.28) is obtained using the Eqs. (3.243.26) [6]. The operators $\Delta=s_{D} S_{0}, \Delta_{c t}=s_{D} S_{c t}^{(n)}$, and $\delta S_{c t}^{(n)} / \delta K_{\Phi}$ are all induced by evanescent operators, although they are not fully evanesced by themselves. The remaining contributions to the $\mathscr{S}_{D}\left(\Gamma_{D R e g}\right)$ cancel. Therefore, in the procedure to find the breaking of the BRST symmetry $\Delta_{\text {breaking }}^{B R S T}$, one can avoid the calculation of the complete renormalized action and performing action of the 4-dimensional Slavnov-Taylor operator $\mathscr{S}$ on it, $\mathscr{S} \Gamma_{r e n}$. Instead, one can evaluate only the contributions from the RHS of Eq. (3.28), and then perform the limit $\mathrm{LIM}_{v \rightarrow 0}$,

$$
\Delta_{b r e a k i n g}^{B R S T}=\operatorname{LIM}_{v \rightarrow 0}\left(\Delta \cdot \Gamma_{D R e g}+\Delta_{c t} \cdot \Gamma_{D R e g}+\int d^{D} x\left[\frac{\delta S_{c t}^{(n)}}{\delta K_{\Phi}(x)} \cdot \Gamma_{D R e g}\right] \frac{\delta \Gamma_{D R e g}}{\delta \Phi(x)}\right) .
$$

Since all operators on the RHS of Eq. (3.29) are induced by evanescent tree-level operator, one can apply the Eq. (3.22) to evaluate them.

For example, at one loop level, this equation can be reexpressed as [6]

$$
\Delta_{\text {breaking }}^{B R S,(1)}=\operatorname{LIM}_{v \rightarrow 0}\left\{[\hat{\Delta}]_{\text {sing }}^{(1)}+b_{D} S_{\text {sct }}^{(1)}\right\}+\left\{\left[N\left[\hat{\Delta} \cdot \Gamma_{\text {ren }}\right]^{(1)}+b S_{\text {fct }}^{(1)}\right\}\right.
$$

where $b_{D}$ and $b$ are linearized Slavnov-Taylor operators $[28,6]$ in $D$ and 4 dimensions respectively. The first curly-bracket term on the RHS of (3.30) appears to be equal to zero. The second term comprises a local finite contribution $N\left[\hat{\Delta} \cdot \Gamma_{r e n}\right]^{(1)}$ which can be evaluated using Bonneau identities. The finite conterterm $b S_{f c t}^{(1)}$ contributuin is needed to restore the BRST symmetry.

\section{Conclusion}

This paper gives a short recapitulation of the Breitenlohner-Maison-'t Hooft-Veltman (BMHV) scheme for dimensional regularization and an overview of the Bonneu identities. In the exposition of the BMHV scheme we reexpressed the Breitenlohner-Maison (BM) Proposition 3 in several ways so as to bridge the notational differences between the BM [5] and Bonneau [1, 2] papers and in order to give a clearer interpretation of the BM renormalization procedure. Specifically, the Bonneau $\bigcirc$ operator is identified to correspond to the procedure of the BM Proposition 3. The explanation of the source of divergences is covered with a corresponding reference [24] and the interpretation of the counterterm operator is supplemented with additional explanations. Further, 
the Lemma 2 of Bonneau is proved in subsection 3.2. in terms of a clearer expression of the BM Proposition 3 as well as an extension of action of the $U_{\gamma}$ operator, appearing in the BM Lemma 5, to the complete amplitudes of subdiagrams and not only to the polynomial form structures coresponding to counterterms. The basic Bonneau identity originally derived for the $\phi^{4}$ theory is rederived for a general theory, and the expression for the 1PI matrix elements is rewritten in terms of the effective action. The quantum numbers of the $v \mathscr{O}_{\delta}$ operator and the operators in terms of which $v \mathscr{O}_{\delta}$ is expressed are identified and the dimensions of these operators are discussed. Two examples of its application have been given: the Bonneau identity for the trace anomaly is rederived for a general theory, for the general special vertex with any number of Lorentz indices larger or equal two. We stated that the trace anomaly covers all the spurious anomalies of the theory. The expression for the 1PI matrix elements is rewritten in terms of the effective action. Finally, we showed how these results may be applied to the evaluation of the breaking of BRST symmetry in dimensionaly regularized theories.

\section{Acknowledgments}

The authors highly acknowledge the financial support from the Croatian Science Foundation (HRZZ) under the project "PRECIOUS" ("Precise Computations of Physical Observables in Supersymmetric Models") number HRZZ-IP-2016-06-7460, as well as the hospitality of the Institut für Kern- und Teilchenphysik, TU Dresden, where part of this work has been pursued. In addition A. Ilakovac highly acknowledges the financial support of the previous Croatian Science Foundation project HRZZ-IP-2013-11-8799. A. Ilakovac and H. Belusca kindly thank the organizers of the Corfu 2019 "Workshop on Connecting Insights in Fundamental Physics: Standard Model and Beyond" for inviting them to present their work. A. Ilakovac higly acknowledges the COST action "Connecting insights in fundamental physics (FUNDAMENTAL CONNECTIONS)" number CA15108 for partial cover of their travel and lodging expences during the workshop. H. Belusca highly acknowledges COST action number CA16201 for the conference grant for the workshop.

\section{References}

[1] G. Bonneau, Zimmermann Identities And Renormalization Group Equation In Dimensional Renormalization, Nucl. Phys. B 167 (1980) 261.

[2] G. Bonneau, Trace and Axial Anomalies in Dimensional Renormalization Through Zimmermann Like Identities, Nucl. Phys. B 171 (1980) 477.

[3] J. Lowenstein, Differential vertex operations in Lagrangian field theory, Commun. Math. Phys. 24 (1971) 1.

[4] Y.-M. P. Lam, Perturbation Lagrangian theory for scalar fields: Ward-Takahasi identity and current algebra, Phys. Rev. D 6 (1972) 2145.

[5] P. Breitenlohner and D. Maison, Dimensional Renormalization and the Action Principle, Commun. Math. Phys. 52 (1977) 11.

[6] C. Martin and D. Sanchez-Ruiz, Action principles, restoration of BRS symmetry and the renormalization group equation for chiral nonAbelian gauge theories in dimensional renormalization with a nonanticommuting gamma(5), Nucl. Phys. B 572 (2000) 387 [hep-th/ 9905076 ]. 
[7] G. 't Hooft and M. Veltman, Regularization and Renormalization of Gauge Fields, Nucl. Phys. B 44 (1972) 189.

[8] P. A. Grassi, T. Hurth and M. Steinhauser, Practical algebraic renormalization, Annals Phys. 288 (2001) 197 [hep-ph/9907426].

[9] P. Grassi, T. Hurth and M. Steinhauser, The Algebraic method, Nucl. Phys. B 610 (2001) 215 [hep-ph/0102005].

[10] I. Fischer, W. Hollik, M. Roth and D. Stockinger, Restoration of supersymmetric Slavnov-Taylor and Ward identities in presence of soft and spontaneous symmetry breaking, Phys. Rev. D 69 (2004) 015004 [hep-ph/0310191].

[11] N. Nakanishi, Graph theory and Feynman integrals, vol. 11 of Mathematics and Its Applications. Gordon and Breach, New York, 1971.

[12] E. R. Speer, Generalized Feynman amplitudes, vol. 62 of Annals of Mathematical Studies. Princeton University Press, 1969.

[13] Y. Haxhimusa, The Structurally Optimal Dual Graph Pyramid and its Application in Image Partitioning, vol. 308 of Disertations in Artificial Inteligence. IOS Press, Amsterdam, Netherlands, 2007.

[14] W. Zimmermann, Convergence of Bogolyubov's method of renormalization in momentum space, Commun. Math. Phys. 15 (1969) 208.

[15] S. D. Deser, M. T. Grisaru and H. Pendleton, eds., Proceedings, 13th Brandeis University Summer Institute in Theoretical Physics, Lectures On Elementary Particles and Quantum Field Theory: Waltham, MA, USA, June 15 - July 24 1970, (Cambridge, MA, USA), MIT, 1970.

[16] J. C. Collins, Renormalization: An Introduction to Renormalization, The Renormalization Group, and the Operator Product Expansion, vol. 26 of Cambridge Monographs on Mathematical Physics. Cambridge University Press, Cambridge, 1986, 10.1017/CBO9780511622656.

[17] K. Symanzik, Dispersion Relations and Vertex Properties in Perturbation Theory, Prog.Theor.Phys 20 (1958) 690.

[18] C. DeWitt and R. Stora, eds., Mécanique statistique et théorie quantique des champs: Proceedings, Ecole d'Eté de Physique Théorique, Les Houches, France, July 5-August 29, 1970, vol. 20 of Les Houches Summer School, (New York, NY), Gordon and Breach, 1971.

[19] S. L. Adler, Axial vector vertex in spinor electrodynamics, Phys. Rev. 177 (1969) 2426.

[20] J. Bell and R. Jackiw, A PCAC puzzle: $\pi^{0} \rightarrow \gamma \gamma$ in the $\sigma$ model, Nuovo Cim. A 60 (1969) 47.

[21] F. Dyson, The S matrix in quantum electrodynamics, Phys. Rev. 75 (1949) 1736.

[22] G. Velo and A. Wightman, eds., Renormalization Theory. Proceedings, NATO Advanced Study Institute: Erice, 17-31 August, 1975, vol. 23 of NATO ASI, C - Mathematical and Physical Sciences, (Dordrecht), Springer, 1976. 10.1007/978-94-010-1490-8.

[23] G. 't Hooft, Dimensional regularization and the renormalization group, Nucl. Phys. B 61 (1973) 455.

[24] G. E. Gel'fand I. M., Shilov, Generalized functions: Volume 1: Properties and operations. Academic Press, New York, 1964.

[25] K. Hepp, Proof of the Bogolyubov-Parasiuk theorem on renormalization, Commun. Math. Phys. 2 (1966) 301. 
[26] J. C. Collins, Normal Products in Dimensional Regularization, Nucl. Phys. B 92 (1975) 477.

[27] H. Bélusca-Maïto, A. Ilakovac, M. Mađor-Božinović and D. Stöckinger, Dimensional Regularization and Breitenlohner-Maison / 't Hooft-Veltman Scheme for $\gamma_{5}$ applied to Chiral YM Theories: Full One-Loop Counterterm and RGE Structure, 2004.14398.

[28] O. Piguet and S. Sorella, Algebraic renormalization: Perturbative renormalization, symmetries and anomalies, vol. 28. 1995, 10.1007/978-3-540-49192-7. 